\title{
Explicit fundamental solutions of some second order differential operators on Heisenberg groups
}

\author{
Isolda Cardoso \\ ECEN-FCEIA, Universidad Nacional de Rosario \\ Pellegrini 250, 2000 Rosario, Argentina \\ E-mail: isolda@fceia.unr.edu.ar \\ Linda Saal \\ FAMAF, Universidad Nacional de Córdoba \\ Ciudad Universitaria, 5000 Córdoba, Argentina \\ E-mail:saal@mate.uncor.edu
}

\begin{abstract}
Let $p, q, n$ be natural numbers such that $p+q=n$. Let $\mathbb{F}$ be either $\mathbb{C}$, the complex numbers field, or $\mathbb{H}$, the quaternionic division algebra. We consider the Heisenberg group $N(p, q, \mathbb{F})$ defined as $N(p, q, \mathbb{F})=\mathbb{F}^{n} \times \mathfrak{I m} \mathbb{F}$, with group law given by

$$
(v, \zeta)\left(v^{\prime}, \zeta^{\prime}\right)=\left(v+v^{\prime}, \zeta+\zeta^{\prime}-\frac{1}{2} \mathfrak{I m} B\left(v, v^{\prime}\right)\right)
$$

where $B(v, w)=\sum_{j=1}^{p} v_{j} \overline{w_{j}}-\sum_{j=p+1}^{n} v_{j} \overline{w_{j}}$. Let $U(p, q, \mathbb{F})$ be the group of $n \times n$ matrices with coefficients in $\mathbb{F}$ that leave invariant the form $B$. In this work we compute explicit fundamental solutions of some second order differential operators on $N(p, q, \mathbb{F})$ which are canonically associated to the action of $U(p, q, \mathbb{F})$.
\end{abstract}

\section{Introduction and Preliminaries}

Let $p, q, n$ be natural numbers such that $p+q=n$. Let $\mathbb{F}$ be either $\mathbb{C}$, the complex numbers field, or $\mathbb{H}$, the quaternionic division algebra. We consider the Heisenberg group $N(p, q, \mathbb{F})$ defined as $N(p, q, \mathbb{F})=\mathbb{F}^{n} \times \mathfrak{I m} \mathbb{F}$, with group law given by

$$
(v, \zeta)\left(v^{\prime}, \zeta^{\prime}\right)=\left(v+v^{\prime}, \zeta+\zeta^{\prime}-\frac{1}{2} \mathfrak{I m} B\left(v, v^{\prime}\right)\right),
$$

where $B(v, w)=\sum_{j=1}^{p} v_{j} \overline{w_{j}}-\sum_{j=p+1}^{n} v_{j} \overline{w_{j}}$. The associated Lie algebra is $\eta(p, q, \mathbb{F})=\mathbb{F}^{n} \oplus \Im \mathfrak{m}(\mathbb{F})$, with Lie bracket given by

$$
\left[(v, \zeta),\left(v^{\prime}, \zeta^{\prime}\right)\right]=\left(0,-\mathfrak{I m} B\left(v, v^{\prime}\right)\right)
$$

2000 Mathematics Subject Classification: Primary 43A80; Secondary 35A08.

Key words and phrases: Heisenberg group, spherical distributions, fundamental solution.

This research was partly supported by research grants form SCyT-UNR and Secyt-UNC, and a fellowship from CONICET. 
Let $\mathcal{U}(\eta(p, q, \mathbb{F}))$ be the universal envelopping algebra of $\eta(p, q, \mathbb{F})$ which we identify with the algebra of left invariant differential operators. Let $U(p, q, \mathbb{H})$ be the group of $n \times n$ matrices with coefficients in $\mathbb{F}$ that leave invariant the form $B$. Then $U(p, q, \mathbb{F})$ acts by automorphism on $\eta(p, q, \mathbb{F})$ by

$$
g \cdot(v, \zeta)=(g v, \zeta)
$$

We denote by $\mathcal{U}(\eta(p, q, \mathbb{F}))^{U(p, q, \mathbb{F})}$ the subalgebra of $\mathcal{U}(\eta(p, q, \mathbb{F}))$ of the left invariant differential operators which commute with this action. It is known that this subalgebra is generated by two operators: $L$ and $U$, and a family of tempered joint eigendistributions is computed explicitly (see for example [D-M], [G-S(1)], [V]).

More precisely, if $\mathbb{F}=\mathbb{C}$ and $\left\{X_{1}, \ldots, X_{n}, Y_{1}, \ldots, Y_{n}, U\right\}$ denotes the standard basis of the Heisenberg Lie algebra with $\left[X_{i}, Y_{j}\right]=\delta_{i j} U$ and all the other brackets are zero, then

$$
L=\sum_{j=1}^{p} X_{j}^{2}+Y_{j}^{2}-\sum_{j=p+1}^{n} X_{j}^{2}+Y_{j}^{2} .
$$

For $\lambda \in \mathbb{R}, \lambda \neq 0$ and $k \in \mathbb{Z}, S_{\lambda, k}$ is a $U(p, q)$-invariant tempered distribution satisfying

$$
\begin{aligned}
L S_{\lambda, k} & =-|\lambda|(2 k+p-q) S_{\lambda, k} \\
i U S_{\lambda, k} & =\lambda S_{\lambda, k} .
\end{aligned}
$$

This family provides us an inversion formula: for all $f$ in the Schwartz space on the Heisenberg group, we have that

$$
f(z, t)=\sum_{k \in \mathbb{Z}} \int_{-\infty}^{\infty} f * S_{\lambda, k}|\lambda|^{n} d \lambda, \quad(z, t) \in N(p, q, \mathbb{C}) .
$$

If $\mathbb{F}=\mathbb{H}$ we take $\left\{X_{1}^{0}, X_{1}^{1}, X_{1}^{2}, X_{1}^{3}, \ldots, X_{n}^{0}, X_{n}^{1}, X_{n}^{2}, X_{n}^{3}, Z_{1}, Z_{2}, Z_{3}\right\}$ the canonical basis for the Lie algebra, where $Z_{1}, Z_{2}, Z_{3}$ generate the center of $\eta(p, q, \mathbb{H})$. Here,

$$
\begin{aligned}
L & =\sum_{r=1}^{p} \sum_{l=0}^{3}\left(X_{r}^{l}\right)^{2}-\sum_{r=p+1}^{n} \sum_{l=0}^{3}\left(X_{r}^{l}\right)^{2}, \quad \text { and } \\
U & =\sum_{l=1}^{3} Z_{l}^{2} .
\end{aligned}
$$

There also exists a family of $U(p, q, \mathbb{H})$-invariant tempered distributions $\varphi_{w, k}, w \in \mathbb{R}^{3}$ y $k \in \mathbb{Z}$, such that each one of them is a joint eigendistribution of $L$ and $U$ :

$$
\begin{aligned}
L \varphi_{w, k} & =-|w|(2 k+2(p-q)) \varphi_{w, k}, \\
U \varphi_{w, k} & =-\lambda^{2} \varphi_{w, k}
\end{aligned}
$$

in this case this family also provides an inversion formula: for all $f \in \mathcal{S}(N(p, q, \mathbb{H}))$ we have that

$$
f(\alpha, z)=\sum_{k \in \mathbb{Z}_{\mathbb{R}^{3}}}\left(f * \varphi_{w, k}\right)(\alpha, z)|w|^{2 n} d w, \quad(\alpha, z) \in N(p, q, \mathbb{H}) .
$$

The aim of this work is to explicitly compute a fundamental solution in the classical case for the operator $\mathcal{L}_{\alpha}=L+i \alpha U$, where $\alpha$ is a complex number; and in the quaternionic case for the operator $L$. Recall that a fundamental solution for the differential operator $\mathcal{L}$ is a tempered distribution $\Phi$ such 
that for all $f$ in the Schwartz class, we have that $\mathcal{L}(f * \Phi)=(\mathcal{L} f) * \Phi=f * \mathcal{L}(\Phi)=f$. So if we define the operator $K$ as $K f=f * \Phi$, then $K \circ \mathcal{L} f=\mathcal{L} \circ K f=f$.

From the inversion formula (1.1) it is natural to propose as a fundamental solution of $\mathcal{L}_{\alpha}$

$$
<\Phi_{\alpha}, f>=\sum_{k \in \mathbb{Z}_{-\infty}} \int_{-\infty}^{\infty} \frac{1}{-|\lambda|(2 k+p-q-\alpha \operatorname{sgn} \lambda)}<S_{\lambda, k}, f>|\lambda|^{n} d \lambda,
$$

for $f \in \mathcal{S}(N(p, q, \mathbb{C}))$; and from (1.2) we propose as a fundamental solution of $L$

$$
<\Phi, f>=\sum_{k \in \mathbb{Z}_{\mathbb{R}^{3}}} \int_{-|w|(2 k+2(p-q))}^{1}<\varphi_{w, k}, f>|w|^{2 n} d w,
$$

for $f \in \mathcal{S}(N(p, q, \mathbb{H}))$.

We remark that for $q=0, \mathbb{F}=\mathbb{C}$ we recover the fundamental solution for the operator $\mathcal{L}_{\alpha}$ given in $[\mathrm{F}-\mathrm{S}]$, and for $q=0, \mathbb{F}=\mathbb{H}$ we recover Kaplan's fundamental solution for the operator $L$ given in $[\mathrm{K}]$. The case $q \neq 0, \alpha=0$ was obtained in [G-S(2)].

The expression of $\Phi_{\alpha}$ is obtained in theorem 2.9, and for the computation we follow the method used in [G-S(2)]. In the quaternionic case, $\Phi$ is given in theorem 3.1, and for its computation we use the Radon transform in order to reduce this case to the classical one.

To describe both families of eigendistributions $\left\{S_{\lambda, k}\right\}$ and $\left\{\varphi_{w, k}\right\}$ we need to adapt a result by Tengstrand in $[\mathrm{T}]$. We describe the elements for the case $\mathbb{F}=\mathbb{C}$, the other one is similar. First of all we take bipolar coordinates on $\mathbb{C}^{n}$ for $\left(x_{1}, y_{1}, \ldots, x_{n}, y_{n}\right)$ we set $\tau=\sum_{j=1}^{p}\left(x_{j}^{2}+y_{j}^{2}\right)-\sum_{j=p+1}^{n}\left(x_{j}^{2}+y_{j}^{2}\right)$, $\rho=\sum_{j=1}^{n}\left(x_{j}^{2}+y_{j}^{2}\right), u=\left(x_{1}, y_{1}, \ldots, x_{p}, y_{p}\right), v=\left(x_{p+1}, y_{p+1}, \ldots, x_{n}, y_{n}\right)$. Hence $u=\left(\frac{\rho+\tau}{2}\right)^{\frac{1}{2}} \omega_{u}$, with $\omega_{u} \in S^{2 p-1}$ and $v=\left(\frac{\rho-\tau}{2}\right)^{\frac{1}{2}} \omega_{v}$, with $\omega_{v} \in S^{2 q-1}$. It is easy to see by changing variables that

$$
\begin{aligned}
\int_{\mathbb{C}^{n}} f(z) d z & =\int_{-\infty}^{\infty} \int_{\rho>|\tau|} \int_{S^{2 p-q} \times S^{2 q-1}} f\left(\left(\frac{\rho+\tau}{2}\right)^{\frac{1}{2}} \omega_{u},\left(\frac{\rho-\tau}{2}\right)^{\frac{1}{2}} \omega_{v}\right) d \omega_{u} d \omega_{v} \times \\
& \times(\rho+\tau)^{p-1}(\rho-\tau)^{q-1} d \rho d \tau .
\end{aligned}
$$

Then we define for $f \in \mathcal{S}\left(\mathbb{R}^{2 n}\right)$

$$
M f(\rho, \tau)=\int_{S^{2 p-1} \times S^{2 q-1}} f\left(\left(\frac{\rho+\tau}{2}\right)^{\frac{1}{2}} \omega_{u},\left(\frac{\rho-\tau}{2}\right)^{\frac{1}{2}} \omega_{v}\right) d \omega_{u} d \omega_{v}
$$

and also

$$
N f(\tau)=\int_{|\tau|}^{\infty} M f(\rho, \tau)(\rho+\tau)^{p-1}(\rho-\tau)^{q-1} d \rho .
$$

Let us now define the space $\mathcal{H}_{n}$ of the functions $\varphi: \mathbb{R} \rightarrow \mathbb{C}$ such that $\varphi(\tau)=\varphi_{1}(\tau)+\tau^{n-1} \varphi_{2}(\tau) H(\tau)$, for $\varphi_{1}, \varphi_{2} \in \mathcal{S}(\mathbb{R})$, where $H$ denotes the Heaviside function. In $[\mathrm{T}]$ it is proved that $\mathcal{H}_{n}$ with a suitable topology is a Fréchet space, and following the same lines we can see that the linear maps $N: \mathcal{S}\left(\mathbb{R}^{2 n}-\{0\}\right) \rightarrow \mathcal{S}(\mathbb{R})$ and $N: \mathcal{S}\left(\mathbb{R}^{2 n}\right) \rightarrow \mathcal{H}$ are continuous and surjective. Let us consider now $\mu \in \mathcal{S}^{\prime}\left(\mathbb{R}^{2 n}\right)^{U(p, q)}$, then it is easy to see that there exists a unique $T \in \mathcal{S}^{\prime}(\mathbb{R})$ such that $<\mu, f>=<$ 
$T, N f>$, for every $f \in \mathcal{S}\left(\mathbb{R}^{2 n}-\{0\}\right)$. Moreover, if $N^{\prime}: \mathcal{H}^{\prime} \rightarrow \mathcal{S}^{\prime}\left(\mathbb{R}^{2 n}\right)$ is the adjoint map, by following again the arguments shown on $[\mathrm{T}]$ we can see that $N^{\prime}$ is a homeomorphism. Finally, for a function $f \in \mathcal{S}(N(p, q, \mathbb{C}))$ we write $N f(\tau, t)$ for $N(f(., t))(\tau)$.

The distributions $S_{\lambda, k}$ are defined as follows

$$
S_{\lambda, k}=\sum_{\left\{m \in \mathbb{N}_{o}^{n}, B(m)=k\right\}} E_{\lambda}\left(h_{m}, h_{m}\right)
$$

where $B(m)=\sum_{j=1}^{p} m_{j}-\sum_{j=p+1}^{n} m_{j}$, the set of functions $\left\{h_{m}\right\} \subset L^{2}\left(\mathbb{R}^{n}\right)$ is the Hermite basis and $E_{\lambda}\left(h, h^{\prime}\right)(z, t)=<\pi_{\lambda}(z, t) h, h^{\prime}>$ are the matrix entries of the Schrödinger representation $\pi_{\lambda}$. Also, $S_{\lambda, k}=e^{-i \lambda t} \otimes F_{\lambda, k}$, where each $F_{\lambda, k} \in \mathcal{S}^{\prime}\left(\mathbb{C}^{n}\right)^{U(p, q)}$ is a tempered distribution defined in terms of the Laguerre polinomials $L_{k}^{m}$ and the map $N$ as follows: for $g \in \mathcal{S}\left(\mathbb{C}^{n}\right), \lambda \neq 0$ and $k \in \mathbb{Z}$

$$
\begin{aligned}
& \text { if } k \geq 0,\left\langle F_{\lambda, k}, g>=<\left(L_{k-q+n-1}^{0} H\right)^{(n-1)}, \tau \rightarrow \frac{2}{|\lambda|} e^{-\frac{\tau}{2}} N g\left(\frac{2}{|\lambda|} \tau\right)>\right.\text {, and } \\
& \text { if } k<0,<F_{\lambda, k}, g>=<\left(L_{-k-p+n-1}^{0} H\right)^{(n-1)}, \tau \rightarrow \frac{2}{|\lambda|} e^{-\frac{\tau}{2}} N g\left(-\frac{2}{|\lambda|} \tau\right)>
\end{aligned}
$$

For the quaternionic case we consider the Schrödinger representation $\pi_{w}$ as given in $[\mathrm{R}]$ (see also $[\mathrm{K}-\mathrm{R}])$ :

$$
\pi_{w}(\alpha, z)=\pi_{|w|}\left(\alpha,<z, \frac{w}{|w|}>\right)
$$

where $\pi_{|w|}$ is the Schrödinger representation for the classical Heisenberg group $N(2 p, 2 q, \mathbb{C})$. We have analogously that the distributions $\varphi_{w, k}$ are defined by

$$
\varphi_{w, k}=\sum_{m \in \mathbb{N}_{0}^{2 n}: B(m)=k} E_{w}\left(h_{m}, h_{m}\right)
$$

where $B(m)=\sum_{j=1}^{2 p} m_{j}-\sum_{j=2 p+1}^{2 n} m_{j}$ and $E_{w}\left(h, h^{\prime}\right)(\alpha, z)=<\pi_{w}(\alpha, z) h, h^{\prime}>$ are the matrix entries of the Schrödinger representation $\pi_{w}$. Moreover, we have that $\varphi_{w, k}=e^{i<w, z>} \otimes \theta_{w, k}$, where $\theta_{w, k}$ is a tempered distribution such that $\theta_{w, k}=N^{\prime} T_{|w|, k}$. If we set $\lambda=|w|$, we have that $T_{|w|, k}=F_{\lambda, k}$ where we replace $n, p, q$ by $2 n, 2 p, 2 q$, respectively, in (1.6) and (1.7). Observe that if we define

$$
\varphi_{\lambda, k}(\alpha, z)=\int_{S^{2}} e^{i<z, \lambda \xi>} d \xi \theta_{\lambda, k}(\alpha),
$$

this distributions are $\operatorname{Spin}(3) \otimes U(p, q, \mathbb{H})$ - invariant.

\section{A fundamental solution for the operator $\mathcal{L}_{\alpha}$}

We have that $\Phi_{\alpha}$ defined as in (1.3) is a well defined tempered distribution, and a fundamental solution for $\mathcal{L}_{\alpha}$. We include the proof since a misprint in Lemma 1 of [M-R] is used in the proof Lemma 2.10 of $[\mathrm{B}-\mathrm{D}-\mathrm{R}]$.

We will consider $\alpha \in \mathbb{C}$ such that $2 k+p-q \pm \alpha \neq 0$ for all $k \in \mathbb{Z}$.

Theorem 2.1. $\Phi_{\alpha}$ defined as in (1.3) is a well defined tempered distribution and it is a fundamental solution for the operator $\mathcal{L}_{\alpha}$. 
Proof. From (1.3) and (1.5) we can write

$$
\begin{array}{r}
\left|<\Phi_{\alpha}, f>\right| \leq \sum_{k \in \mathbb{Z}} \int_{0}^{\infty}\left(\left|\frac{<S_{-\lambda, k}, f>}{(2 k+p-q+\alpha)}\right|+\left|\frac{<S_{\lambda, k}, f>}{(2 k+p-q-\alpha)}\right|\right)|\lambda|^{n-1} d \lambda \leq \\
\quad \leq \sum_{k \in \mathbb{Z}} \int_{0}^{\infty} \sum_{\substack{\beta \in \mathbb{N}_{0}^{n} \\
B(\beta)=k}}^{\infty}\left(\left|\frac{<E_{-\lambda}\left(h_{\beta}, h_{\beta}\right), f>}{(2 k+p-q+\alpha)}\right|+\left|\frac{<E_{\lambda}\left(h_{\beta}, h_{\beta}\right), f>}{(2 k+p-q-\alpha)}\right|\right)|\lambda|^{n-1} d \lambda .
\end{array}
$$

From the known facts that $\sum_{k \in \mathbb{Z}} \sum_{\substack{\beta \in \mathbb{N}_{0}^{n} \\ B(\beta)=k}} p(\beta)=\sum_{k \geq 0}\left(\begin{array}{c}k+n-1 \\ n-1\end{array}\right) p(k)$,

$\left|<E_{\lambda}\left(h_{\beta}, h_{\beta}\right), f>\right|=\left|<\pi_{\lambda}(f) h_{\beta}, h_{\beta}>\right| \leq\|f\|_{L^{1}(N(p, q, \mathbb{C}))}$ and that for $m \in \mathbb{N}$

$$
\pi_{\lambda}(f) h_{\beta}=\frac{1}{(-1)^{m}|\lambda|^{m}(2 B(\beta)+p-q+\alpha \operatorname{sgn}(\lambda))^{m}} \pi_{\lambda}\left(L^{m} f\right) h_{\beta}
$$

we get that

$$
\begin{aligned}
\mid & <\Phi_{\alpha}, f>\mid \leq\left\|L^{m} f\right\|_{L^{1}(N(p, q, \mathbb{C}))} \times \\
& \times \sum_{k \geq 0} \int_{0}^{\infty}\left(\begin{array}{c}
k+n-1 \\
k
\end{array}\right)\left(\frac{|\lambda|^{n-1-m}}{|2 k+p-q+\alpha|^{m+1}}+\frac{|\lambda|^{n-1-m}}{|2 k+p-q-\alpha|^{m+1}}\right) d \lambda .
\end{aligned}
$$

Let us consider the first term, the second one is analogous. We split the integral between $|\lambda| \mid 2 k+p-$ $q+\alpha \mid \geq 1$ and $0 \leq|\lambda||2 k+p-q+\alpha| \leq 1$.Thus,

$$
\sum_{k \geq 0}\left(\begin{array}{c}
k+n-1 \\
k
\end{array}\right) \int_{|\lambda||2 k+p-q+\alpha| \geq 1} \frac{1}{|2 k+p-q+\alpha|^{m+1}}|\lambda|^{n-1-m} d \lambda
$$

is finite if we take $m>n$, and

$$
\sum_{k \geq 0}\left(\begin{array}{c}
k+n-1 \\
k
\end{array}\right) \int_{0 \leq|\lambda||2 k+p-q+\alpha| \leq 1} \frac{1}{|2 k+p-q+\alpha|^{m+1}}|\lambda|^{n-1-m} d \lambda
$$

is finite for $m<n$. From the above computations it also follows that $\Phi_{\alpha}$ is a tempered distribution. Next we see that it is a fundamental solution by writing $L=L_{0}+L_{1}$, which in coordinates are

$$
\begin{aligned}
L_{0}= & \frac{1}{4}\left(\sum_{j=1}^{p}\left(x_{j}^{2}+y_{j}^{2}\right)-\sum_{j=p+1}^{n}\left(x_{j}^{2}+y_{j}^{2}\right)\right) \frac{\partial^{2}}{\partial t^{2}}+ \\
& +\sum_{j=1}^{p}\left(\frac{\partial^{2}}{\partial x_{j}^{2}}+\frac{\partial^{2}}{\partial y_{j}^{2}}\right)-\sum_{j=p+1}^{n}\left(\frac{\partial^{2}}{\partial x_{j}^{2}}+\frac{\partial^{2}}{\partial y_{j}^{2}}\right), \\
L_{1}= & \frac{\partial}{\partial t} \sum_{j=1}^{n}\left(x_{j} \frac{\partial}{\partial y_{j}}-y_{j} \frac{\partial}{\partial x_{j}}\right) .
\end{aligned}
$$

Then, as $L_{0}, L_{1}$ and $T$ commute with left translations and also $L_{0}\left(g^{\vee}\right)=\left(L_{0} g\right)^{\vee}, L_{1}\left(g^{\vee}\right)=-\left(L_{1} g\right)^{\vee}$ and $T\left(g^{\vee}\right)=-(T g)^{\vee}$ we get that

$$
\left(\mathcal{L} f * \Phi_{\alpha}\right)(z, t)=<\Phi_{\alpha},\left(L_{(z, t)^{-1}} \mathcal{L} f\right)^{\vee}>=<\Phi_{\alpha},\left(L_{0}-i \alpha\right)\left(L_{(z, t)^{-1}} f\right)^{\vee},
$$


because $L_{1} \Phi_{\alpha}=0$. Hence,

$$
\begin{gathered}
\left(\mathcal{L}_{\alpha} f * \Phi\right)(z, t)=\sum_{k \in \mathbb{Z}} \int_{-\infty}^{\infty} \frac{<S_{\lambda, k},\left(L_{0}-i \alpha T\right)\left(L_{(z, t)^{-1}} f\right)^{\vee}>}{-|\lambda|(2 k+p-q-\alpha \operatorname{sgn} \lambda)}|\lambda|^{n-1} d \lambda= \\
=\sum_{k \in \mathbb{Z}} \int_{-\infty}^{\infty} \frac{<\left(L_{0}+i \alpha T\right) S_{\lambda, k},\left(L_{(z, t)^{-1}} f\right)^{\vee}>}{-|\lambda|(2 k+p-q-\alpha \operatorname{sgn} \lambda)}|\lambda|^{n-1} d \lambda= \\
=\sum_{k \in \mathbb{Z}} \int_{-\infty}^{\infty}<S_{\lambda, k},\left(L_{(z, t)^{-1}} f\right)^{\vee}>|\lambda|^{n-1} d \lambda=f(z, t),
\end{gathered}
$$

because of the inversion formula. The other one, $f * \mathcal{L}_{\alpha}(f)=f$, is immediate.

Now we proceed with the computation of $\Phi_{\alpha}$. Given that the series (1.3) defining $\Phi_{\alpha}$ converges absolutely, we can split the sum over $k \in \mathbb{Z}$ into the sums for $k \geq q$, for $k \leq-p$ and for $-p<k<q$. In the first case we change the summation index writing $k=k^{\prime}+q$ and in the second as well, writing $k=k^{\prime}-p$. So we get

$$
\begin{aligned}
& <\Phi_{\alpha}, f>=(-1) \sum_{k^{\prime} \geq 0} \frac{1}{2 k^{\prime}+n-\alpha} \int_{0}^{\infty}\left[<S_{\lambda, k^{\prime}+q}, f>-<S_{\lambda,-k^{\prime}-p}, f>\right]|\lambda|^{n-1} d \lambda+ \\
& \quad+(-1) \sum_{k^{\prime} \geq 0} \frac{1}{2 k^{\prime}+n+\alpha} \int_{0}^{\infty}\left[<S_{-\lambda, k^{\prime}+q}, f>-<S_{-\lambda,-k^{\prime}-p}, f>\right]|\lambda|^{n-1} d \lambda+ \\
& \quad+(-1) \sum_{-p<k<q} \int_{0}^{\infty}\left(\frac{<S_{-\lambda, k}, f>}{2 k+p-q+\alpha}+\frac{<S_{\lambda, k}, f>}{2 k+p-q-\alpha}\right)|\lambda|^{n-1} d \lambda .
\end{aligned}
$$

By Abel's Lemma and the Lebesgue dominated convergence theorem we can write $\Phi_{\alpha}=\Phi_{1}+\Phi_{2}$ where

$$
\begin{aligned}
<\Phi_{1}, f> & =\lim _{r \rightarrow 1^{-}} \lim _{\epsilon \rightarrow 0^{+}}(-1) \sum_{k^{\prime} \geq 0} \frac{r^{2 k^{\prime}+n-\alpha}}{2 k^{\prime}+n-\alpha} \int_{0}^{\infty} e^{-\epsilon|\lambda|} \times \\
& \times\left[<S_{\lambda, k^{\prime}+q}, f>-<S_{\lambda,-k^{\prime}-p}, f>\right]|\lambda|^{n-1} d \lambda+ \\
+ & \lim _{r \rightarrow 1^{-}} \lim _{\epsilon \rightarrow 0^{+}}(-1) \sum_{k^{\prime} \geq 0} \frac{r^{2 k^{\prime}+n+\alpha}}{2 k^{\prime}+n+\alpha} \int_{0}^{\infty} e^{-\epsilon|\lambda|} \times \\
\times & {\left[<S_{-\lambda, k^{\prime}+q}, f>-<S_{-\lambda,-k^{\prime}-p}, f>\right]|\lambda|^{n-1} d \lambda, } \\
<\Phi_{2}, f> & =\lim _{\epsilon \rightarrow 0^{+}}(-1) \sum_{-p<k<q} \int_{0}^{\infty} e^{-\epsilon|\lambda|} \times \\
& \times\left(\frac{<S_{-\lambda, k}, f>}{2 k+p-q+\alpha}+\frac{<S_{\lambda, k}, f>}{2 k+p-q-\alpha}\right)|\lambda|^{n-1} d \lambda .
\end{aligned}
$$


Using that $S_{\lambda, k}=e^{-i \lambda t} \otimes F_{\lambda, k}$ and the computations from [G-S(2)], namely (2.6) to (2.9), we get that

$$
\begin{aligned}
& <\Phi_{1}, f>=\lim _{r \rightarrow 1^{-}} \lim _{\epsilon \rightarrow 0^{+}}(-1) \sum_{k \geq 0} \frac{r^{2 k+n-\alpha}}{2 k+n-\alpha} \int_{0}^{\infty} e^{-\epsilon|\lambda|} \int_{-\infty}^{\infty} e^{-i \lambda t} \times \\
& \times<\left(L_{k+n-1}^{0} H\right)^{(n-1)}, \frac{2}{|\lambda|} e^{-\frac{\tau}{2}}\left[N f\left(\frac{2}{|\lambda|} \tau, t\right)-N f\left(-\frac{2}{|\lambda|} \tau, t\right)\right]>d t d \lambda+ \\
& +\lim _{r \rightarrow 1^{-}} \lim _{\epsilon \rightarrow 0^{+}}(-1) \sum_{k \geq 0} \frac{r^{2 k+n+\alpha}}{2 k+n+\alpha} \int_{0}^{\infty} e^{-\epsilon|\lambda|} \int_{-\infty}^{\infty} e^{i \lambda t} \times \\
& \quad \times<\left(L_{k+n-1}^{0} H\right)^{(n-1)}, \frac{2}{|\lambda|} e^{-\frac{\tau}{2}}\left[N f\left(\frac{2}{|\lambda|} \tau, t\right)-N f\left(-\frac{2}{|\lambda|} \tau, t\right)\right]>d t d \lambda,
\end{aligned}
$$

and setting

$$
b_{k, l}=\sum_{j=l}^{n-2}\left(\begin{array}{l}
j \\
l
\end{array}\right)\left(\frac{1}{2}\right)^{2-l}(-1)^{n-j}\left(\begin{array}{l}
k+n-1 \\
n-j-2
\end{array}\right)
$$

we have that

$$
\begin{aligned}
& <\Phi_{1}, f>=\lim _{r \rightarrow 1^{-}} \lim _{\epsilon \rightarrow 0^{+}} \sum_{k \geq 0} \frac{r^{2 k+n-\alpha}}{2 k+n-\alpha} \int_{0}^{\infty} e^{-\epsilon|\lambda|} \int_{-\infty}^{\infty} e^{-i \lambda t} \times \\
& \times\left[(-1)^{n} \int_{-\infty}^{\infty} L_{k}^{n-1}\left(\frac{|\lambda|}{2}|s|\right) e^{-\frac{|\lambda|}{4}|s|} \operatorname{sgn}(s) N f(s, t) d s+\right. \\
& \left.-2 \sum_{\substack{l=0 \\
l \text { odd }}}^{n-2}\left(\frac{2}{|\lambda|}\right)^{l+1} b_{k, l} \frac{\partial^{l} N f}{\partial \tau^{l}}(0, t)\right] d t d \lambda+ \\
& +\lim _{r \rightarrow 1^{-}} \lim _{\epsilon \rightarrow 0^{+}} \sum_{k \geq 0} \frac{r^{2 k+n+\alpha}}{2 k+n+\alpha} \int_{0}^{\infty} e^{-\epsilon|\lambda|} \int_{-\infty}^{\infty} e^{i \lambda t} \times \\
& \times\left[(-1)^{n} \int_{-\infty}^{\infty} L_{k}^{n-1}\left(\frac{|\lambda|}{2}|s|\right) e^{-\frac{|\lambda|}{4}|s|} \operatorname{sgn}(s) N f(s, t) d s+\right. \\
& \left.-2 \sum_{\substack{l=0 \\
l \text { odd }}}^{n-2}\left(\frac{2}{|\lambda|}\right)^{l+1} b_{k, l} \frac{\partial^{l} N f}{\partial \tau^{l}}(0, t)\right] d t d \lambda .
\end{aligned}
$$

Now we define

$$
G_{f}(\tau, t)=N f(\tau, t)-\sum_{j=0}^{n-2} \frac{\partial^{j} N f}{\partial \tau^{j}}(0, t) \frac{\tau^{j}}{j !},
$$

and then we can split $\Phi_{1}=\Phi_{11}+\Phi_{12}$ where 


$$
\begin{aligned}
& <\Phi_{11}, f>=\lim _{r \rightarrow 1^{-}} \lim _{\epsilon \rightarrow 0^{+}} \sum_{k \geq 0}(-1)^{n} \frac{r^{2 k+n-\alpha}}{2 k+n-\alpha} \int_{0}^{\infty} \int_{-\infty}^{\infty} e^{-\epsilon|\lambda|} e^{-i \lambda t}|\lambda|^{n-1} \times \\
& \times \int_{-\infty}^{\infty} L_{k}^{n-1}\left(\frac{|\lambda|}{2}|\tau|\right) e^{-\frac{|\lambda|}{4}|\tau|} \operatorname{sgn}(\tau) G_{f}(\tau, t) d \tau d t d \lambda+ \\
& +\lim _{r \rightarrow 1^{-}} \lim _{\epsilon \rightarrow 0^{+}} \sum_{k \geq 0}(-1)^{n} \frac{r^{2 k+n+\alpha}}{2 k+n+\alpha} \int_{0}^{\infty} \int_{-\infty}^{\infty} e^{-\epsilon|\lambda|} e^{i \lambda t}|\lambda|^{n-1} \times \\
& \times \int_{-\infty}^{\infty} L_{k}^{n-1}\left(\frac{|\lambda|}{2}|\tau|\right) e^{-\frac{|\lambda|}{4}|\tau|} \operatorname{sgn}(\tau) G_{f}(\tau, t) d \tau d t d \lambda \\
& <\Phi_{12}, f>=\lim _{r \rightarrow 1^{-}} \lim _{\epsilon \rightarrow 0^{+}} \sum_{k \geq 0} \frac{r^{2 k+n-\alpha}}{2 k+n-\alpha} \int_{0}^{\infty} \int_{-\infty}^{\infty} e^{-\epsilon|\lambda|} e^{-i \lambda t}|\lambda|^{n-1} \times \\
& \times 2 \sum_{\substack{l=0 \\
l \text { odd }}}^{n-2}\left(\frac{2}{|\lambda|}\right)^{l+1}\left(a_{k, l}+b_{k, l}\right) \frac{\partial^{l} N f}{\partial \tau^{l}}(0, t) d t d \lambda+ \\
& +\lim _{r \rightarrow 1^{-}} \lim _{\epsilon \rightarrow 0^{+}} \sum_{k \geq 0} \frac{r^{2 k+n+\alpha}}{2 k+n+\alpha} \int_{0}^{\infty} \int_{-\infty}^{\infty} e^{-\epsilon|\lambda|} e^{i \lambda t}|\lambda|^{n-1} \times \\
& \times 2 \sum_{\substack{l=0 \\
l \text { odd }}}^{n-2}\left(\frac{2}{|\lambda|}\right)^{l+1}\left(a_{k, l}+b_{k, l}\right) \frac{\partial^{l} N f}{\partial \tau^{l}}(0, t) d t d \lambda,
\end{aligned}
$$

with

$$
a_{k, l}=(-1)^{n} \frac{1}{l !} \int_{0}^{\infty} L_{k}^{n-1}(s) e^{-\frac{s}{2}} s^{l} d s .
$$

We will show that $\Phi_{11}$ is well defined. We have proved that the series (1.3) defining $\Phi_{\alpha}$ converges and as $\Phi_{2}$ is a finite sum we will obtain that $\Phi_{12}$ is also well defined.

Proposition 2.2. The following identities hold:

(i)

$$
\begin{aligned}
& \int_{-\infty}^{\infty} e^{-\epsilon|\lambda|} e^{-i \lambda t} L_{k}^{n-1}\left(\frac{|\lambda|}{2}|\tau|\right) e^{-\frac{|\lambda|}{4}|\tau|}|\lambda|^{n-1} d \lambda= \\
& \quad=4^{n}(n-1) !(-1)^{n}\left(\begin{array}{c}
k+n-1 \\
k
\end{array}\right)\left(\frac{(|\tau|-4 \epsilon-4 i t)^{k}}{(|\tau|+4 \epsilon+4 i t)^{k+n}}\right) .
\end{aligned}
$$


(ii)

$$
\begin{aligned}
& \lim _{\epsilon \rightarrow 0^{+}} \int_{\mathbb{R}^{2}}\left(\frac{(|\tau|-4 i t-4 \epsilon)^{k}}{(|\tau|+4 i t+4 \epsilon)^{k+n}}\right) \operatorname{sgn}(\tau) G_{f}(\tau, t) d \tau d t= \\
& \quad=\int_{\mathbb{R}^{2}} \frac{1}{(|\tau|-4 i t)^{\frac{n}{2}-\frac{\alpha}{2}}} \frac{1}{(|\tau|+4 i t)^{\frac{n}{2}+\frac{\alpha}{2}}}\left(\frac{|\tau|-4 i t}{\tau^{2}+16 t^{2}}\right)^{2 k+n-\alpha} \operatorname{sgn}(\tau) G_{f}(\tau, t) d \tau d t .
\end{aligned}
$$

(iii)

$$
\begin{aligned}
& \lim _{\epsilon \rightarrow 0^{+}} \int_{\mathbb{R}^{2}}\left(\frac{(|\tau|+4 i t-4 \epsilon)^{k}}{(|\tau|-4 i t+4 \epsilon)^{k+n}}\right) \operatorname{sgn}(\tau) G_{f}(\tau, t) d \tau d t= \\
& \quad=\int_{\mathbb{R}^{2}} \frac{1}{(|\tau|-4 i t)^{\frac{n}{2}-\frac{\alpha}{2}}} \frac{1}{(|\tau|+4 i t)^{\frac{n}{2}+\frac{\alpha}{2}}}\left(\frac{|\tau|-4 i t}{\tau^{2}+16 t^{2}}\right)^{2 k+n+\alpha} \operatorname{sgn}(\tau) G_{f}(\tau, t) d \tau d t .
\end{aligned}
$$

Proof. From (4.9) of [G-S(2)] we know that (i) follows from the generating identity for the Laguerre polynomials:

$$
\sum_{k \geq 0} L_{k}^{n-1}(t) z^{k}=\frac{1}{(1-z)^{n}} e^{-\frac{z t}{1-z}}
$$

From Lemma 2.2 of $[\mathrm{G}-\mathrm{S}(2)]$, which states that the function $\frac{G_{f}(\tau, t)}{\left(\tau^{2}+16 t^{2}\right)^{\frac{n}{2}}}$ is integrable in $\mathbb{R}^{2}$ and the fact that $\left|\frac{1}{(|\tau|-4 i t)^{-\frac{\alpha}{2}}}\right|\left|\frac{1}{(|\tau|+4 i t)^{\frac{\alpha}{2}}}\right|=1$, it follows that the function $\frac{1}{(|\tau|-4 i t)^{\frac{n}{2}-\frac{\alpha}{2}}} \frac{1}{(|\tau|+4 i t)^{\frac{n}{2}+\frac{\alpha}{2}}} G_{f}(\tau, t)$ is integrable in $\mathbb{R}^{2}$. So we get (ii). For (iii) we just change $e^{-i \lambda t}$ by $e^{i \lambda t}$ and argue like for (ii).

Then, by Proposition 2.2,

$$
\begin{aligned}
&<\Phi_{11}, f>=\beta_{n} \lim _{r \rightarrow 1^{-}} \sum_{k \geq 0} \frac{r^{2 k+n-\alpha}}{2 k+n-\alpha} \alpha_{k} \times \\
& \times \int_{\mathbb{R}^{2}}\left(\frac{|\tau|-4 i t}{\tau^{2}+16 t^{2}}\right)^{2 k+n-\alpha} \frac{\operatorname{sgn}(\tau) G_{f}(\tau, t)}{(|\tau|-4 i t)^{\frac{n}{2}-\frac{\alpha}{2}}(|\tau|+4 i t)^{\frac{n}{2}+\frac{\alpha}{2}}} d \tau d t+ \\
&+\beta_{n} \lim _{r \rightarrow 1^{-}} \sum_{k \geq 0} \frac{r^{2 k+n+\alpha}}{2 k+n+\alpha} \alpha_{k} \times \\
& \quad \int_{\mathbb{R}^{2}}\left(\frac{|\tau|+4 i t}{\tau^{2}+16 t^{2}}\right)^{2 k+n+\alpha} \frac{\operatorname{sgn}(\tau) G_{f}(\tau, t)}{(|\tau|+4 i t)^{\frac{n}{2}+\frac{\alpha}{2}}(|\tau|-4 i t)^{\frac{n}{2}-\frac{\alpha}{2}}} d \tau d t,
\end{aligned}
$$

where $\beta_{n}=4^{n}(n-1) !(-1)^{n}$ y $\alpha_{k}=\left(\begin{array}{c}k+n-1 \\ k\end{array}\right)(-1)^{k}$.

To study $\left\langle\Phi_{11}, f>\right.$ we split each integral over the left and right halfplanes and take polar 
coordinates $\tau-4 i t=\rho e^{i \theta}$ to obtain

$$
\begin{aligned}
<\Phi_{11}, f> & \beta_{n} \lim _{r \rightarrow 1^{-}} \sum_{k \geq 0} \alpha_{k} \frac{r^{2 k+n-\alpha}}{2 k+n-\alpha} \times \\
\times & \int_{0}^{\infty}\left[\int_{-\frac{\pi}{2}}^{\frac{\pi}{2}} e^{i(2 k+n-\alpha) \theta} \frac{1}{4 \rho^{n-1}} e^{i \alpha \theta} \operatorname{sgn}(\cos \theta) G_{f}\left(\rho \cos \theta,-\frac{\rho}{4} \sin \theta\right) d \theta+\right. \\
& \left.+\int_{\frac{\pi}{2}}^{\frac{3}{2} \pi} \frac{e^{-i(2 k+n-\alpha) \theta} e^{-i \alpha \theta}}{(-1)^{n} 4 \rho^{n-1}} \operatorname{sgn}(\cos \theta) G_{f}\left(\rho \cos \theta,-\frac{\rho}{4} \sin \theta\right) d \theta\right] d \rho+ \\
+\beta_{n} \lim _{r \rightarrow 1^{-}} \sum_{k \geq 0} \alpha_{k} \frac{r^{2 k+n+\alpha}}{2 k+n+\alpha} \times & \int_{0}^{\infty}\left[\int_{-\frac{\pi}{2}}^{\frac{\pi}{2}} e^{-i(2 k+n+\alpha) \theta} \frac{1}{4 \rho^{n-1}} e^{i \alpha \theta} \operatorname{sgn}(\cos \theta) G_{f}\left(\rho \cos \theta,-\frac{\rho}{4} \sin \theta\right) d \theta+\right. \\
& \left.+\int_{\frac{\pi}{2}}^{\frac{3}{2} \pi} \frac{e^{i(2 k+n+\alpha) \theta} e^{-i \alpha \theta}}{(-1)^{n} 4 \rho^{n-1}} \operatorname{sgn}(\cos \theta) G_{f}\left(\rho \cos \theta,-\frac{\rho}{4} \sin \theta\right) d \theta\right] d \rho
\end{aligned}
$$

Now we change variable in the second and fourth term according to $\theta \longleftrightarrow-\theta$. Then, in the fourth term we change variables again according to $\theta \longleftrightarrow \theta+2 \pi$. By proposition 2.2 we can interchange the integration order, so we can write

$$
\begin{aligned}
<\Phi_{11}, f> & =\beta_{n} \lim _{r \rightarrow 1^{-}} \int_{0}^{\infty} \int_{-\frac{\pi}{2}}^{\frac{\pi}{2}} e^{i \alpha \theta} \times \\
\times & {\left[\sum_{k \geq 0} \alpha_{k}\left(\frac{r^{2 k+n-\alpha}}{2 k+n-\alpha} e^{i(2 k+n-\alpha) \theta}+\frac{r^{2 k+n+\alpha}}{2 k+n+\alpha} e^{-i(2 k+n+\alpha) \theta}\right)\right] \times } \\
\times & \frac{1}{\rho^{n-1}} \operatorname{sgn}(\cos \theta) G_{f}\left(\rho \cos \theta,-\frac{\rho}{4} \sin \theta\right) d \theta d \rho+ \\
+ & \frac{(-1)^{n}}{4} \beta_{n} \lim _{r \rightarrow 1^{-}} \int_{0}^{\infty} \int_{\frac{\pi}{2}}^{\frac{3}{2} \pi} e^{i \alpha \theta} \times \\
& \times\left[\sum_{k \geq 0} \alpha_{k}\left(\frac{r^{2 k+n-\alpha}}{2 k+n-\alpha} e^{i(2 k+n-\alpha) \theta}+\frac{r^{2 k+n+\alpha}}{2 k+n+\alpha} e^{-i(2 k+n+\alpha) \theta}\right)\right] \times \\
& \times \frac{1}{\rho^{n-1}} \operatorname{sgn}(\cos \theta) G_{f}\left(\rho \cos \theta, \frac{\rho}{4} \sin \theta\right) d \theta d \rho .
\end{aligned}
$$

Let $I$ denote the real interval $\left[-\frac{\pi}{2}, \frac{\pi}{2}\right]$. Next let us consider the vector space

$$
\mathcal{X}=\left\{g \in C^{n-2}(I): g^{(j)}\left( \pm \frac{\pi}{2}\right)=0,0 \leq j \leq n-2, g^{(n-1)} \in L^{\infty}(I)\right\} .
$$


We identify each function $g \in \mathcal{X}$ with the function $\widetilde{g}$ on $S^{1}=\frac{\mathbb{R}}{\mathbb{Z}}$, defined equal to 0 outside $\operatorname{supp}(g)$ and we make no distinction between $g$ and $\widetilde{g}$. Thus, if $g \in \mathcal{X}$ then $g \in C^{n-2}\left(S^{1}\right)$ with $g^{(n-1)} \in$ $L^{\infty}\left(S^{1}\right)$. Observe that if $g \in \mathcal{X}$, then also $e^{i \alpha \theta} g \in \mathcal{X}$. The topology on $\mathcal{X}$ is given by $\|g\|_{\mathcal{X}}=$ $\max _{0 \leq j \leq n-1}\left\|g^{(j)}\right\|_{\infty}$.

For $k \in \mathbb{Z}$ we set $\alpha_{k}=\left(\begin{array}{c}k+n-1 \\ k\end{array}\right)(-1)^{k}$. Now let us define

$$
\begin{gathered}
\Psi_{r, \alpha}(\theta)=\sum_{k \geq 0} \alpha_{k}\left(\frac{r^{2 k+n-\alpha} e^{i(2 k+n-\alpha) \theta}}{2 k+n-\alpha}+\frac{r^{2 k+n+\alpha} e^{-i(2 k+n+\alpha) \theta}}{2 k+n+\alpha}\right), \\
<\Psi_{\alpha}, g>=<\sum_{k \geq 0} \alpha_{k}\left(\frac{e^{i(2 k+n-\alpha) \theta}}{2 k+n-\alpha}+\frac{e^{-i(2 k+n+\alpha) \theta}}{2 k+n+\alpha}\right), g>,
\end{gathered}
$$

and let us see that $\Psi_{\alpha} \in \mathcal{X}^{\prime}$, the dual space of $\mathcal{X}$. Indeed,

$$
\left|<\Psi_{\alpha}, g>\right| \leq\left|e^{i \alpha \theta}\right| \sum_{k \geq 0}\left(\begin{array}{c}
k+n-1 \\
k
\end{array}\right)\left(\frac{\left|<e^{i(2 k+n) \theta}, g>\right|}{|2 k+n-\alpha|}+\mid \frac{<e^{-i(2 k+n) \theta}, g>\mid}{|2 k+n+\alpha|}\right) .
$$

If $\widehat{g}(n)=<g, e^{i n \theta}>$ denotes the $n$-th Fourier coefficient of $g$, then we have that

$$
\begin{aligned}
\mid<\Psi_{\alpha}, g & >\mid \leq c \sum_{k \geq 0} \frac{k^{n-1}}{|2 k+n|^{n-1}}\left(\frac{\left|\widehat{g^{(n-1)}}(2 k+n)\right|}{|2 k+n-\alpha|}+\frac{\left|\widehat{g^{(n-1)}}(-2 k-n)\right|}{|2 k+n+\alpha|}\right) \leq \\
& \leq c \sum_{k \geq 0} \frac{1}{k} \widehat{\mid g^{(n-1)}}(2 k+n)\left|+\frac{1}{k}\right| \widehat{g^{(n-1)}}(-2 k-n) \mid \leq \\
& \leq c\left(\sum_{k \geq 0} \frac{1}{k^{2}}\right)^{\frac{1}{2}}\left|\widehat{g^{(n-1)}}\right|_{L^{2}},
\end{aligned}
$$

where we used the Cauchy-Schwarz inequality. Observe that the constants $c$ are not the same on each expression.

By Abel's Lemma, $\lim _{r \rightarrow 1^{-}} \Psi_{r, \alpha}=\Psi_{\alpha}$ in $\mathcal{X}^{\prime}$ that is, with respect to the weak convergence topology. Similarly, if $J$ denotes the real interval $\left[\frac{\pi}{2}, \frac{3}{2} \pi\right]$, we define the space,

$$
\mathcal{Y}=\left\{g \in C^{n-2}(J): g^{(j)}\left(\frac{\pi}{2}\right)=g^{(j)}\left(\frac{3}{2} \pi\right)=0,0 \leq j \leq n-2, g^{(n-1)} \in L^{\infty}(J)\right\},
$$

and we obtain that $\Psi_{\alpha}$ is well defined in $\mathcal{Y}^{\prime}$ and $\lim _{r \rightarrow 1^{-}} \Psi_{r, \alpha}=\Psi_{\alpha}$ in $\mathcal{Y}^{\prime}$.

Our aim now is to compute $\Psi_{\alpha}$.

From Proposition 3.7 of $[\mathrm{G}-\mathrm{S}(2)]$ we know that if $\Theta \in \mathcal{D}^{\prime}\left(S^{1}\right)$ is defined by

$$
\Theta(\theta)=i \sum_{k \geq 0}\left(\begin{array}{c}
k+n-1 \\
k
\end{array}\right)(-1)^{k} e^{i(2 k+n) \theta},
$$

then for even $n$ we have that

$$
\mathfrak{R e} \Theta(\theta)=\frac{d}{d \theta} Q_{n-2}\left(\frac{d}{d \theta}\right)\left(\delta_{\frac{\pi}{2}}+\delta_{-\frac{\pi}{2}}\right)=\sum_{j=0}^{n-2} c_{j}\left(\delta_{\frac{\pi}{2}}^{(j+1)}+\delta_{-\frac{\pi}{2}}^{(j+1)}\right),
$$

where $Q_{n-2}$ is a polynomial of degree $n-2$; and for odd $n$ we have that

$$
\mathfrak{R e} \Theta(\theta)=d_{0} \frac{d}{d \theta} \widetilde{H}+\frac{d}{d \theta} Q_{n-2}\left(\frac{d}{d \theta}\right)\left(\delta_{\frac{\pi}{2}}-\delta_{-\frac{\pi}{2}}\right)=d_{0}\left(\delta_{-\frac{\pi}{2}}-\delta_{\frac{\pi}{2}}\right)+\sum_{j=0}^{n-2} c_{j}\left(\delta_{\frac{\pi}{2}}^{(j+1)}-\delta_{-\frac{\pi}{2}}^{(j+1)}\right),
$$


where $Q_{n-2}$ is a polynomial of degree $n-2$, and $\widetilde{H}(\theta)=H(\cos \theta)$.

Let us recall the generating identity for the Laguerre polynomials (2.9), and take $t=0$ and $z=-r^{2} e^{2 i \theta}$. We get

$$
\sum_{k \geq 0}\left(\begin{array}{c}
k+n-1 \\
k
\end{array}\right)(-1)^{k} r^{2 k+n} e^{i(2 k+n) \theta}=\left(\frac{r e^{i \theta}}{1+r^{2} e^{2 i \theta}}\right)^{n} .
$$

We also need a couple of results:

Lemma 2.3. For a fixed $r>1$ the functions $\alpha \rightarrow \Psi_{r, \alpha}(0)$ and $\alpha \rightarrow \lim _{r \rightarrow 1^{-}} \Psi_{r, \alpha}(0)$ are analytic on $\Omega=\mathbb{C} \backslash F$, where $F=\{2 k+n: k \in \mathbb{Z}\}$.

Proof. Let $K$ be a compact set, $K \subset \Omega$. It is easy to see that for fixed $r$ the series (2.10) converges uniformly, since

$$
\left|\Psi_{r, \alpha}(0)\right| \leq \max _{\alpha \in K}\left|r^{\alpha}\right|\left(\frac{r}{1+r^{2}}\right)^{n} d(K, F) .
$$

Also, for $\alpha \in \Omega$ there exists $\lim _{r \rightarrow 1^{-}} \Psi_{r, \alpha}(0)$. Indeed, if $0 \leq r_{1}<r<r_{2}<1$, from the Mean Value Theorem we have that for some $\xi \in\left(r_{1}, r_{2}\right)$,

$$
\begin{aligned}
& \Psi_{r_{1}, \alpha}(0)-\Psi_{r_{2}, \alpha}(0)=\frac{d}{d r} \Psi_{\xi, \alpha}(0)\left(r_{2}-r_{1}\right)=\left(\xi^{-\alpha-1}+\xi^{\alpha-1}\right) \sum_{k \geq 0} \alpha_{k} \xi^{2 k+n}\left(r_{2}-r_{1}\right)= \\
& =\left(\xi^{-\alpha-1}+\xi^{\alpha-1}\right)\left(\frac{\xi}{1+\xi^{2}}\right)^{n}\left(r_{2}-r_{1}\right),
\end{aligned}
$$

where the last equality holds from (2.16). Hence

$$
\left|\Psi_{r_{1}, \alpha}(0)-\Psi_{r_{2}, \alpha}(0)\right| \leq c(\xi)\left|r_{2}-r_{1}\right|,
$$

where $c(\xi)$ is a constant which depends on $\xi$. Moreover, for $\alpha \in K$ and $\xi \in\left[\frac{1}{2}, 1\right], \xi^{n-\alpha-1}+\xi^{n+\alpha-1}$ is bounded in $K \times\left[\frac{1}{2}, 1\right]$, so the convergence is uniform, hence $\alpha \rightarrow \lim _{r \rightarrow 1^{-}} \Psi_{r, \alpha}(0)$ is an analytic function.

Lemma 2.4. Let $0<\delta<\frac{\pi}{4}$. For $0<r<1$ and $0 \leq|\theta|<\delta$ we have that

$$
\left|\Psi_{r, \alpha}(\theta)-\Psi_{r, \alpha}(0)\right| \leq\left(\max _{0 \leq|\theta|<\delta} e^{|\mathfrak{I m} \alpha||\theta|}\right)\left(a\left|r^{-\alpha}-r^{\alpha}\right|+b\left|r^{\alpha}\right|(1-r)\right)|\theta|,
$$

with $a, b$ positive constants. Also for $0 \leq|\theta-\pi|<\delta<\frac{\pi}{4}$,

$$
\left|\Psi_{r, \alpha}(\theta)-\Psi_{r, \alpha}(\pi)\right| \leq\left(\max _{0 \leq|\theta-\pi|<\delta} e^{|\mathfrak{I} \mathfrak{m} \alpha||\theta|}\right)\left(a\left|r^{-\alpha}-r^{\alpha}\right|+b\left|r^{\alpha}\right|(1-r)\right)|\theta-\pi|,
$$

with $a, b$ positive constants.

Proof. We will estimate $\left|\Psi_{r, \alpha}(\theta)-\Psi_{r, \alpha}(0)\right|$ for $0<|\theta|<\delta<\frac{\pi}{4}$, the other case is similar. We have

$$
\begin{gathered}
\frac{d}{d \theta} \Psi_{r, \alpha}(\theta)=i e^{-i \alpha \theta} \sum_{k \geq 0} \alpha_{k} r^{2 k+n}\left(\left(r^{-\alpha}-r^{\alpha}\right) e^{i(2 k+n) \theta}+\left(e^{i(2 k+n) \theta}-e^{-i(2 k+n) \theta}\right) r^{\alpha}\right)= \\
=i e^{-i \alpha \theta}\left(\left(r^{-\alpha}-r^{\alpha}\right)\left(\frac{r e^{i \theta}}{1+r^{2} e^{2 i \theta}}\right)^{n}+2 i r^{\alpha} \mathfrak{I m}\left(\frac{r e^{i \theta}}{1+r^{2} e^{i 2 \theta}}\right)^{n}\right)
\end{gathered}
$$


because of (2.16). We have that

$$
\left|\frac{d}{d \theta} \Psi_{r, \alpha}(\theta)\right| \leq e^{|\mathfrak{I m} \alpha||\theta|}\left(\left|r^{-\alpha}-r^{\alpha}\right|\left|\left(\frac{r e^{i \theta}}{1+r^{2} e^{i 2 \theta}}\right)^{n}\right|+2\left|r^{\alpha}\right|\left|\mathfrak{I m}\left(\frac{r e^{i \theta}}{1+r^{2} e^{i 2 \theta}}\right)^{n}\right|\right) .
$$

From Proposition 3.1 of $[\mathrm{G}-\mathrm{S}(2)]$ we know that $\left|\mathfrak{I m}\left(\frac{r e^{i \theta}}{1+r^{2} e^{i 2 \theta}}\right)^{n}\right| \longrightarrow 0$ as $r \rightarrow 1^{-}$, uniformly for $|\theta|<\frac{\pi}{4},|\theta-\pi|<\frac{\pi}{4}$. Also, $\left|\left(\frac{r e^{i \theta}}{1+r^{2} e^{i 2 \theta}}\right)^{n}\right| \leq c$, for a constant $c$. Then $\left|\frac{d}{d \theta} \Psi_{r, \alpha}(\theta)\right| \longrightarrow 0$ uniformly on $|\theta|<\frac{\pi}{4}$ as $r \rightarrow 1^{-}$, and we get the desired inequality by applying the Mean Value Theorem around 0 .

Now we can state the following

Proposition 2.5. For $f \in \mathcal{X}$ we have that $\left\langle\Psi_{\alpha}, f>=C_{\alpha}<1, f>\right.$, where $C_{\alpha}=\frac{\Gamma\left(\frac{n+\alpha}{2}\right) \Gamma\left(\frac{n-\alpha}{2}\right)}{(n-1) !}$; and for $f \in \mathcal{Y}$ we have that $\left\langle\Psi_{\alpha}, f>=\widetilde{C_{\alpha}}<1, f>\right.$, where $\widetilde{C_{\alpha}}=(-1)^{n} e^{-i \alpha \pi} C_{\alpha}$.

Proof. First we consider $f \in \mathcal{X}$ such that $\int_{-\frac{\pi}{2}}^{\frac{\pi}{2}} f(t) d t=0$ and we define $F(\theta)=\int_{-\frac{\pi}{2}}^{\theta} f(t) d t$. It is easy to see that $F \in \mathcal{X}$ and $F^{\prime}=f$. Because of the integration by parts formula we have that

$$
\begin{aligned}
<\Psi_{\alpha}, f> & =<\Psi_{\alpha}, F^{\prime}>=\int_{-\frac{\pi}{2}}^{\frac{\pi}{2}} \sum_{k \geq 0} \alpha_{k}\left(\frac{e^{i(2 k+n-\alpha) \theta}}{2 k+n-\alpha}+\frac{e^{-i(2 k+n+\alpha) \theta}}{2 k+n+\alpha}\right) F^{\prime}(\theta) d \theta= \\
& =-<\Theta, e^{-i \alpha \theta} F>-<\bar{\Theta}, e^{-i \alpha \theta} F>
\end{aligned}
$$

where $\bar{\Theta}=\sum_{k \geq 0}\left(\begin{array}{c}k+n-1 \\ k\end{array}\right)(-1)^{k} e^{-i(2 k+n) \theta}$. So if $n$ is even, from (2.14) we get that

$$
<\Psi_{\alpha}, f>=-\sum_{j=0}^{n-2} c_{j}<\delta_{\frac{\pi}{2}}^{(j+1)}+\delta_{\frac{-\pi}{2}}^{(j+1)}, e^{-i \alpha \theta} F>-\sum_{j=0}^{n-2} \overline{c_{j}}<\overline{\delta_{\frac{\pi}{2}}^{(j+1)}}+\overline{\delta_{\frac{-\pi}{2}}^{(j+1)}}, e^{-i \alpha \theta} F>
$$

and because $<\delta_{ \pm \frac{\pi}{2}}^{(j+1)}, e^{-i \alpha \theta} F>=0$ we conclude that $<\Psi_{\alpha}, f>=0$. If $n$ is odd we use (2.15) to conclude that $\left\langle\Psi_{\alpha}, f\right\rangle=0$.

For the general case of any $f \in \mathcal{X}$ we consider $h \in \mathcal{X}$ such that $\int_{-\frac{\pi}{2}}^{\frac{\pi}{2}} h(t) d t=1$ and define $g(\theta)=f(\theta)-\left(\int_{-\frac{\pi}{2}}^{\frac{\pi}{2}} f(t) d t\right) h(\theta)$. So we can apply the above result to $g$ and get that $\left\langle\Psi_{\alpha}, g\right\rangle=0$. Then $<\Psi_{\alpha}, f>=<\Psi_{\alpha}, g>+<\Psi_{\alpha}, h><1, f>=<\Psi_{\alpha}, h><1, f>$. Let $C_{\alpha}=<\Psi_{\alpha}, h>$.

In order to compute $C_{\alpha}$, consider $g \in \mathcal{X}$ such that $\operatorname{supp}(g) \subset\left(-\frac{\pi}{4}, \frac{\pi}{4}\right), \int_{\frac{-\pi}{4}}^{\frac{\pi}{4}} g(t) d t=1$ and $g \geq 0$ we have that

$$
<e^{i \alpha \theta} \Psi_{\alpha}, g>=C_{\alpha} \int_{\frac{-\pi}{2}}^{\frac{\pi}{2}} e^{i \alpha \theta} g(\theta) d \theta
$$

and also that

$$
<e^{i \alpha \theta} \Psi_{\alpha}, g>=\lim _{r \rightarrow 1^{-}}\left(\int_{-\frac{\pi}{2}}^{\frac{\pi}{2}}\left(\Psi_{r, \alpha}(\theta)-\Psi_{r, \alpha}(0)\right) e^{i \alpha \theta} g(\theta) d \theta+\Psi_{r, \alpha}(0) \int_{-\frac{\pi}{2}}^{\frac{\pi}{2}} e^{i \alpha \theta} g(\theta) d \theta\right) .
$$


From lemmas 1 and 2 we get that

$$
C_{\alpha}=\lim _{r \rightarrow 1^{-}} \Psi_{r, \alpha}(0)
$$

and also that $C_{\alpha}$ is an analytic function of $\alpha$. Given that $\Psi_{0, \alpha}(0)=0$ we can write

$$
C_{\alpha}=\lim _{r \rightarrow 1^{-}} \Psi_{r, \alpha}(0)=\Psi_{1, \alpha}(0)-\Psi_{0, \alpha}(0)=\int_{0}^{1} w_{\alpha}^{\prime}(s) d s
$$

where

$$
w_{\alpha}(r)=\Psi_{r, \alpha}(0)=r^{-\alpha} \sum_{k \geq 0} \alpha_{k} \frac{r^{2 k+n}}{2 k+n-\alpha}+r^{\alpha} \sum_{k \geq 0} \alpha_{k} \frac{r^{2 k+n}}{2 k+n+\alpha} .
$$

Applying (2.9) with $\theta=0$ we obtain $w_{\alpha}^{\prime}(r)=\left(r^{-\alpha-1}+r^{\alpha-1}\right) \sum_{k \geq 0} \alpha_{k} r^{2 k+n}=\left(r^{-\alpha-1}+r^{\alpha-1}\right)\left(\frac{r}{1+r^{2}}\right)^{n}$, and we can solve the integral for $\mathfrak{R e}(n+\alpha)>0, \mathfrak{R e}(n-\alpha)>0$, getting

$$
C_{\alpha}=B\left(\frac{n+\alpha}{2}, \frac{n-\alpha}{2}\right)=\frac{\Gamma\left(\frac{n+\alpha}{2}\right) \Gamma\left(\frac{n-\alpha}{2}\right)}{(n-1) !}
$$

where $B$ is the Beta function and $\Gamma$ is the Gamma function. By lemma 2.3, (2.18) holds for the other range of $\alpha$, by analytic continuation. In a completely analogous way we get that $\widetilde{C_{\alpha}}=(-1)^{n} e^{-i \alpha \pi} C_{\alpha}$.

Let us now define

$$
K_{1 f}(\rho, \theta)=\frac{1}{\rho^{n-1}} \operatorname{sgn}(\cos \theta) G_{f}\left(\rho \cos \theta,-\frac{\rho}{4} \sin \theta\right),
$$

for $\theta \in\left[-\frac{\pi}{2}, \frac{\pi}{2}\right], 0<\rho<\infty$, where $G_{f}$ is the function defined in (2.4); and

$$
K_{2 f}(\rho, \theta)=\frac{1}{\rho^{n-1}} \operatorname{sgn}(\cos \theta) G_{f}\left(\rho \cos \theta, \frac{\rho}{4} \sin \theta\right),
$$

for $\theta \in\left[\frac{\pi}{2}, \frac{3}{2} \pi\right], 0<\rho<\infty$.

It is easy to check that $K_{1 f}(\rho,.) \in \mathcal{X}$. Recall that we changed variables according to $\tau-4 i t=\rho e^{i \theta}$. Since $N f \in \mathcal{H}_{n}$, there exists a positive constant $c$ such that

$$
\sup _{\tau \neq 0, t \in \mathbb{R}}\left|\left(\tau^{2}+16 t^{2}\right) N f(\tau, t)\right| \leq c,
$$

that is

$$
\left|N f\left(\rho \cos \theta,-\frac{\rho}{4} \sin \theta\right)\right| \leq \frac{c}{\rho^{2}}
$$

Also since $N f(0,.) \in \mathcal{S}(\mathbb{R})$, there exists a positive constant $c_{N}$ such that for $t \in \mathbb{R}$,

$$
\left|t^{N} \sum_{j=0}^{n-2} \frac{\partial^{j}}{\partial \tau^{j}} N f(0, t) \frac{\tau^{j}}{j !}\right| \leq c_{N}|\tau|^{n-2} .
$$

Thus, for $N \in \mathbb{N}$ there exists $c_{N}$ such that

$$
\left|K_{1 f}(\rho, \theta)\right| \leq \frac{a}{\rho^{n+1}}+\frac{b}{\rho^{N+1}} \frac{|\cos \theta|^{n-2}}{|\sin \theta|^{N}} .
$$

Analogous observations are also true for $K_{2 f}$. 
Proposition 2.6. Let $C_{\alpha}$ and $\widetilde{C_{\alpha}}$ be the constans obtained in (2.18). Let $K_{1 f}$ and $K_{2 f}$ be as defined in (2.19) and (2.20) and $\alpha_{k}=\left(\begin{array}{c}k+n-1 \\ k\end{array}\right)(-1)^{k}$. Then

$$
\begin{aligned}
& \lim _{r \rightarrow 1^{-}} \int_{0}^{\infty} \int_{-\frac{\pi}{2}}^{\frac{\pi}{2}} e^{i \alpha \theta} \sum_{k \geq 0} \alpha_{k}\left(\frac{r^{2 k+n-\alpha} e^{i(2 k+n-\alpha) \theta}}{2 k+n-\alpha}+\frac{r^{2 k+n+\alpha} e^{-i(2 k+n+\alpha) \theta}}{2 k+n+\alpha}\right) K_{1 f}(\rho, \theta) d \theta d \rho= \\
& \quad=4^{n-1}(n-1) ! C_{\alpha} \int_{\mathbb{R}} \int_{\tau>0} \frac{1}{(\tau-4 i t)^{\frac{n-\alpha}{2}}} \frac{1}{(\tau+4 i t)^{\frac{n+\alpha}{2}}} \operatorname{sgn}(\tau) G_{f}(\tau, t) d \tau d t,
\end{aligned}
$$

and

$$
\begin{aligned}
& \lim _{r \rightarrow 1^{-}} \int_{0}^{\infty} \int_{\frac{\pi}{2}}^{\frac{3}{2} \pi} e^{i \alpha \theta} \sum_{k \geq 0} \alpha_{k}\left(\frac{r^{2 k+n-\alpha} e^{i(2 k+n-\alpha) \theta}}{2 k+n-\alpha}+\frac{r^{2 k+n+\alpha} e^{-i(2 k+n+\alpha) \theta}}{2 k+n+\alpha}\right) K_{2 f}(\rho, \theta) d \theta d \rho= \\
& \quad=4^{n-1}(n-1) ! \widetilde{C_{\alpha}} \int_{\mathbb{R}} \int_{\tau<0} \frac{1}{(\tau-4 i t)^{\frac{n-\alpha}{2}}} \frac{1}{(\tau+4 i t)^{\frac{n+\alpha}{2}}} \operatorname{sgn}(\tau) G_{f}(\tau, t) d \tau d t .
\end{aligned}
$$

Proof. The proof follows the same lines that Proposition 4.2 of [G-S(2)]. We only sketch it for completeness.

Taking polar coordinates $\tau-4 i t=\rho e^{i \theta}$ we only need to show that

$$
\lim _{r \rightarrow 1^{-}} \int_{0}^{\infty}<\Psi_{r, \alpha}, e^{i \alpha \theta} K_{1 f}(\rho, \theta)>d \rho=\int_{0}^{\infty}<C_{\alpha}, e^{i \alpha \theta} K_{1 f}(\rho, \theta)>d \rho .
$$

In order to do this we split the integral for $0<\rho<1$ and $1<\rho<\infty$.

We consider first the case $1<\rho<\infty$. For $|\theta| \leq \delta<\frac{\pi}{4}$, set $I=\int_{1}^{\infty} \int_{|\theta|<\delta} e^{i \alpha \theta}\left(\Psi_{r, \alpha}(\theta)-\right.$ $\left.\Psi_{r, \alpha}(0)\right) K_{1 f}(\rho, \theta) d \theta d \rho$ and $I I=\int_{1}^{\infty} \int_{|\theta|<\delta} e^{i \alpha \theta}\left(\Psi_{r, \alpha}(0)-C_{\alpha}\right) K_{1 f}(\rho, \theta) d \theta d \rho$. We bound $I$ close to 0 by applying Lemma 2.4 and taking $N=1$ in (2.21). For $I I$ we just take $N=\frac{1}{2}$ in (2.21). To analize the case $\delta \leq|\theta| \leq \frac{\pi}{2}$, we observe that the function $K_{1 f}^{*}(\theta)=\int_{1}^{\infty} K_{1 f}(\rho, \theta) d \rho$ defined for $\theta \in\left[-\frac{\pi}{2},-\delta\right] \cup\left[\delta, \frac{\pi}{2}\right]$ can be extended to an element of $\mathcal{X}$ that we still denote by $K_{1 f}^{*}$. Then

$$
\begin{aligned}
& \int_{1}^{\infty} \int_{\delta<|\theta|<\frac{\pi}{2}} e^{i \alpha \theta}\left(\Psi_{r, \alpha}(\theta)-C_{\alpha}\right) K_{1 f}(\rho, \theta) d \theta d \rho= \\
& \quad=\int_{-\frac{\pi}{2}}^{\frac{\pi}{2}} e^{i \alpha \theta}\left(\Psi_{r, \alpha}(\theta)-C_{\alpha}\right) K_{1 f}^{*}(\theta) d \theta-\int_{|\theta|<\delta} e^{i \alpha \theta}\left(\Psi_{r, \alpha}(\theta)-C_{\alpha}\right) K_{1 f}^{*}(\theta) d \theta .
\end{aligned}
$$

The first term converges to zero as $r \rightarrow 1^{-}$since $\Psi_{r, \alpha} \rightarrow C_{\alpha}$ as $r \rightarrow 1^{-}$in $\mathcal{X}^{\prime}$. For the second term we argue as above.

Finally, for the case $0<\rho<1$ we apply the same arguments to the function $K_{1 f}^{* *}(\theta)=\int_{0}^{1} K_{1 f}(\rho, \theta) d \rho$. 
Corollary 2.7. $\left\langle\Phi_{11}, f>\right.$ is well defined for $f \in \mathcal{S}\left(\mathbb{H}_{n}\right)$, and

$$
\begin{gathered}
<\Phi_{11}, f>=4^{n-1}(n-1) ! C_{\alpha} \int_{\mathbb{R}} \int_{\tau>0} \frac{1}{(\tau-4 i t)^{\frac{n-\alpha}{2}}} \frac{1}{(\tau+4 i t)^{\frac{n+\alpha}{2}}} \operatorname{sgn}(\tau) G_{f}(\tau, t) d \tau d t+ \\
+4^{n-1}(n-1) ! \widetilde{C_{\alpha}} \int_{\mathbb{R}} \int_{\tau<0} \frac{1}{(\tau-4 i t)^{\frac{n-\alpha}{2}}} \frac{1}{(\tau+4 i t)^{\frac{n+\alpha}{2}}} \operatorname{sgn}(\tau) G_{f}(\tau, t) d \tau d t .
\end{gathered}
$$

From the corollary we also get that $\left\langle\Phi_{12}, f\right\rangle$ is well defined. In order to explicitly compute it we define for $0 \leq l \leq n-2, \epsilon>0$ and $f \in \mathcal{S}\left(\mathbb{H}_{n}\right)$

$$
\begin{gathered}
d_{\epsilon, l, f}^{-}=\int_{0}^{\infty} \int_{-\infty}^{\infty} e^{-\epsilon|\lambda|} e^{-i \lambda t}|\lambda|^{n-l-2} \frac{\partial^{l}}{\partial \tau^{l}} N f(0, t) d t d \lambda, \quad \text { and } \\
d_{\epsilon, l, f}^{+}=\int_{0}^{\infty} \int_{-\infty}^{\infty} e^{-\epsilon|\lambda|} e^{i \lambda t}|\lambda|^{n-l-2} \frac{\partial^{l}}{\partial \tau^{l}} N f(0, t) d t d \lambda .
\end{gathered}
$$

Then we can write $(2.7)$ as

$$
<\Phi_{12}, f>=\lim _{r \rightarrow 1^{-}} \lim _{\epsilon \rightarrow 0^{+}} \sum_{k \geq 0} \sum_{\substack{l=0 \\ l \text { odd }}}^{n-2} 2^{l+2}\left(a_{k l}+b_{k l}\right)\left[\frac{r^{2 k+n-\alpha}}{2 k+n-\alpha} d_{\epsilon, l, f}^{-}+\frac{r^{2 k+n+\alpha}}{2 k+n+\alpha} d_{\epsilon, l, f}^{+}\right] .
$$

From Lemma 4.4 in $[\mathrm{G}-\mathrm{S}(2)]$ we deduce that

$$
a_{k l}+b_{k l}=(-1)^{k} \sum_{j=1}^{l+1} \frac{1}{2^{n-l-j-1}}\left(\begin{array}{c}
n-j-1 \\
l-j+1
\end{array}\right)\left(\begin{array}{c}
j+k-1 \\
k
\end{array}\right) .
$$

Also we have the following

Lemma 2.8. If $0 \leq l \leq n-2, \epsilon>0$ and $f \in \mathcal{S}\left(\mathbb{H}_{n}\right)$, then

$$
\begin{gathered}
\lim _{\epsilon \rightarrow 0^{+}} d_{\epsilon, l, f}^{-}=\frac{1}{i^{n-l-2}}<\frac{\pi}{2} \delta-i(v p)\left(\frac{1}{\lambda}\right), \frac{\partial^{n-2}}{\partial \lambda^{n-l-2} \partial \tau^{l}} N f(0, .)>\quad \text { and } \\
\lim _{\epsilon \rightarrow 0^{+}} d_{\epsilon, l, f}^{+}=i^{n-l-2}<\frac{\pi}{2} \delta+i(v p)\left(\frac{1}{\lambda}\right), \frac{\partial^{n-2}}{\partial \lambda^{n-l-2} \partial \tau^{l}} N f(0, .)>.
\end{gathered}
$$

Proof. Let us consider $g(\lambda)=e^{-\epsilon|\lambda|}|\lambda|^{n-l-2}$ and $h(t)=\frac{\partial^{l}}{\partial \tau^{l}} N f(0, t)$, and observe that $\int_{-\infty}^{\infty} e^{-i \lambda t} h(t) d t=$ $\hat{h}(\lambda)$. Then just by using properties of the Fourier transform we get that

$$
d_{\epsilon, l, f}^{-}=\int_{0}^{\infty} \int_{-\infty}^{\infty} g(\lambda) e^{-i \lambda t} h(t) d t d \lambda=\int_{0}^{\infty} g(\lambda) \hat{h}(\lambda) d \lambda=\frac{1}{i^{n-l-2}} \int_{-\infty}^{\infty} \frac{1}{\epsilon+i \lambda} h^{(n-l-2)}(\lambda) d \lambda .
$$

For each $\epsilon>0, \frac{1}{\epsilon+i \lambda}$ is a distribution such that there exists $\lim _{\epsilon \rightarrow 0^{+}} \frac{1}{\epsilon+i \lambda}$ in $\mathcal{S}^{\prime}(\mathbb{R})$. Moreover, it is easy to check that

$$
\lim _{\epsilon \rightarrow 0^{+}} \frac{1}{\epsilon+i \lambda}=\frac{\pi}{2} \delta-i(v p)\left(\frac{1}{\lambda}\right) .
$$

Thus the desired equality follows. For $d_{\epsilon, l, f}^{+}$we need to change variable according to $\lambda \longleftrightarrow-\lambda$ after considering the Fourier transform of $h$. 
We define for $j \in \mathbb{N}, 0<j<n-1$, the functions of $r$, with $0 \leq r<1$, by

$$
\begin{aligned}
& w_{j}^{-}(r)=\sum_{k \geq 0}(-1)^{k}\left(\begin{array}{c}
j+k-1 \\
k
\end{array}\right) \frac{r^{2 k+n-\alpha}}{2 k+n-\alpha}, \\
& w_{j}^{+}(r)=\sum_{k \geq 0}(-1)^{k}\left(\begin{array}{c}
j+k-1 \\
k
\end{array}\right) \frac{r^{2 k+n+\alpha}}{2 k+n+\alpha} .
\end{aligned}
$$

We can see, in a complete analogous way as the computations made for $C_{\alpha}$ and $\widetilde{C_{\alpha}}$, that this functions are well defined and that

$$
\begin{aligned}
& c_{j}^{-}:=\lim _{r \rightarrow 1^{-}} w_{j}^{-}(r)=\frac{1}{2} B_{\frac{1}{2}}\left(\frac{n-\alpha}{2}, j-\frac{n-\alpha}{2}\right), \text { and } \\
& c_{j}^{+}:=\lim _{r \rightarrow 1^{-}} w_{j}^{+}(r)=\frac{1}{2} B_{\frac{1}{2}}\left(\frac{n+\alpha}{2}, j-\frac{n+\alpha}{2}\right),
\end{aligned}
$$

where $B_{\frac{1}{2}}$ is another special function called the incomplete Beta function.

We now plug all of this definitions and results together to finally obtain an expression for $\Phi_{12}$ :

$$
\begin{aligned}
<\Phi_{12}, f> & =\sum_{\substack{l=0 \\
l \text { odd }}}^{n-2} \sum_{j=1}^{l+1} 2^{2 l-n+j+3}\left(\begin{array}{c}
n-j-1 \\
l-j+1
\end{array}\right)\left[\left(\frac{1}{i^{n-l-2}} c_{j}^{-}+i^{n-l-2} c_{j}^{+}\right) \frac{\pi}{2}\right] \times \\
\times & <\delta, \frac{\partial^{n-2}}{\partial \lambda^{n-l-2} \partial \tau^{l}} N f(0, .)>+ \\
+(-1) & \sum_{l=0}^{n-2} \sum_{j=1}^{l+1} 2^{2 l-n+j+3}\left(\begin{array}{c}
n-j-1 \\
l-j+1
\end{array}\right)\left(\frac{1}{i^{n-l+1}} c_{j}^{-}+i^{n-l+1} c_{j}^{+}\right) \times \\
& \times(v p)\left(\frac{1}{\lambda}\right), \frac{\partial^{n-2}}{\partial \lambda^{n-l-2} \partial \tau^{l}} N f(0, .)>
\end{aligned}
$$

All we need to do now is to use again lemma 2.8 to get an expression for $\Phi_{2}$. Thus, we have proved the following

Theorem 2.9. Let $C_{\alpha}$ and $\widetilde{C_{\alpha}}$ be the constans defined as in (2.18), and let $c_{j}^{-}$and $c_{j}^{+}$the constants 
defined as in (2.25). Then

$$
\begin{aligned}
<\Phi, & >=4^{n-1}(n-1) ! C_{\alpha} \int_{\mathbb{R}} \int_{\tau>0} \frac{1}{(\tau-4 i t)^{\frac{n-\alpha}{2}}} \frac{1}{(\tau+4 i t)^{\frac{n+\alpha}{2}}} \operatorname{sgn}(\tau) G_{f}(\tau, t) d \tau d t+ \\
+ & 4^{n-1}(n-1) ! \widetilde{C_{\alpha}} \int_{\mathbb{R}} \int_{\tau<0} \frac{1}{(\tau-4 i t)^{\frac{n-\alpha}{2}}} \frac{1}{(\tau+4 i t)^{\frac{n+\alpha}{2}}} \operatorname{sgn}(\tau) G_{f}(\tau, t) d \tau d t+ \\
+ & \sum_{\substack{l=0 \\
l \text { odd }}}^{n-2} \sum_{j=1}^{l+1} 2^{2 l-n+j+3}\left(\begin{array}{l}
n-j-1 \\
l-j+1
\end{array}\right)\left[\left(\frac{1}{i^{n-l-2}} c_{j}^{-}+i^{n-l-2} c_{j}^{+}\right) \frac{\pi}{2}\right] \times \\
& \times<\delta, \frac{\partial^{n-2}}{\partial \lambda^{n-l-2} \partial \tau^{l}} N f(0, .)>+ \\
+ & (-1) \sum_{l=0}^{n-2} \sum_{j=1}^{l+1} 2^{2 l-n+j+3}\left(\begin{array}{c}
n-j-1 \\
l-j
\end{array}\right)\left(\frac{1}{i^{n-l+1}} c_{j}^{-}+i^{n-l+1} c_{j}^{+}\right) \times \\
& \times<(v p)\left(\frac{1}{\lambda}\right), \frac{\partial^{n-2}}{\partial \lambda^{n-l-2} \partial \tau^{l}} N f(0, .)>+ \\
+ & \sum_{k=0}^{n-2} \sum_{l=0}^{n-2} c_{k l}\left[\frac{1}{n-2 k+\alpha-2} i^{n-l-2}<\frac{\pi}{2} \delta+i(v p)\left(\frac{1}{\lambda}\right), \frac{\partial^{n-2}}{\partial \lambda^{n-l-2} \partial \tau^{l}} N f(0, .)>+\right. \\
& \left.+\frac{1}{n-2 k-\alpha-2} \frac{1}{i^{n-l-2}}<\frac{\pi}{2} \delta-i(v p)\left(\frac{1}{\lambda}\right), \frac{\partial^{n-2}}{\partial \lambda^{n-l-2} \partial \tau^{l}} N f(0, .)>\right]
\end{aligned}
$$

where $c_{k l}=\sum_{\substack{1 \leq j \leq n-2 \\ j \geq n-k-2}} 2^{2 l-n-j}(-1)^{n-j}\left(\begin{array}{l}j \\ l\end{array}\right)\left(\begin{array}{l}k-l+1 \\ n-j-2\end{array}\right)$.

\section{A fundamental solution for $L$}

Like in the classical case, we have that the distribution $\Phi$ defined in (1.4) is a well defined tempered distribution and it is a relative fundamental solution for the operator $L$. The proof is identical to the one of theorem 2.1.

We will compute the fundamental solution $\Phi$ by means of the Radon transform and the fundamental solution of the operator $L$ in the classical case $\mathbb{H}_{2 n}$.

Let $F \in \mathcal{S}\left(\mathbb{R}^{3}\right)$. We assign to $F$ a function $\mathcal{R} F: \mathbb{R} \times S^{2} \rightarrow \mathbb{R}$ defined by

$$
\mathcal{R} F(t, \xi)=\int_{\mathbb{R}^{2}} F\left(t \xi+u_{1} e_{1}+u_{2} e_{2}\right) d u_{1} d u_{2},
$$

where $\left\{\xi, e_{1}, e_{2}\right\}$ is an orthonormal basis of $\mathbb{R}^{3}$. It is easy to see that this definition does not depend on the choice of the basis. In order to recover $F$ from $\mathcal{R} F$, we consider the space $\mathcal{S}\left(\mathbb{R} \times S^{2}\right)$ of the continuous functions $G: \mathbb{R} \times S^{2} \rightarrow \mathbb{R}$ that are infinitely differentiable in $t$ and satisfy for every $m, k \in \mathbb{N}_{0}$ that

$$
\sup _{t \in \mathbb{R}, \xi \in S^{2}}\left|t^{m} \frac{\partial^{k}}{\partial t^{k}} G(t, \xi)\right|<\infty .
$$

Now for $G \in \mathcal{S}\left(\mathbb{R} \times S^{2}\right)$ we define a function $\mathcal{R}^{*} G: \mathbb{R}^{3} \rightarrow \mathbb{R}$ by

$$
\mathcal{R}^{*} G(z)=\int_{S^{2}} G(<z, \xi>, \xi) d \xi .
$$


Both assignments are well defined, and moreover $\mathcal{R}: \mathcal{S}\left(\mathbb{R}^{3}\right) \rightarrow \mathcal{S}\left(\mathbb{R} \times S^{2}\right)$ is the Radon transform, $\mathcal{R}^{*}: \mathcal{S}\left(\mathbb{R} \times S^{2}\right) \rightarrow \mathcal{S}\left(\mathbb{R}^{3}\right)$ is the dual Radon transform and they satisfy for every $F \in \mathcal{S}\left(\mathbb{R}^{3}\right)$

$$
-2 \pi F=\triangle \mathcal{R}^{*} \mathcal{R} F
$$

where $\triangle=\frac{\partial^{2}}{\partial z_{1}^{2}}+\frac{\partial^{2}}{\partial z_{2}^{2}}+\frac{\partial^{2}}{\partial z_{3}^{2}}$ is the $\mathbb{R}^{3}$-Laplacian.

Now let us consider the function $\phi$ defined for a fixed $\tau, \tau \neq 0$ by

$$
\phi(\tau, z)=\frac{16 n}{\pi} \frac{4^{2 n}(2 n-1) ! c_{0}}{\left(\tau^{2}+16|z|^{2}\right)^{n+1}}
$$

where $c_{0}=-\int_{0}^{1} \sigma^{2 n-1}\left(1+\sigma^{2}\right)^{2 n} d \sigma$. The function $\phi(\tau,$.$) is not a Schwartz function on \mathbb{R}^{3}$, but we have that $(1+\triangle)^{k} \phi(\tau,.) \in L^{1}\left(\mathbb{R}^{3}\right)$, hence $\left(1+|\xi|^{2}\right)^{k} \widehat{\phi(\tau, .)}(\xi) \in L^{\infty}\left(\mathbb{R}^{3}\right)$. With these properties the inversion formula for the Radon transform (3.1) still holds. The proof follows straightforward from theorem 5.4 of $[\mathrm{S}-\mathrm{Sh}]$. Let us now compute the Radon transform of the function $\phi$.

$$
\begin{aligned}
\mathcal{R} \phi(\tau, t, \xi) & =\int_{\mathbb{R}^{2}} \frac{16 n}{\pi} \frac{4^{2 n}(2 n-1) ! c_{0}}{\left(\tau^{2}+16\left(t^{2}+u_{1}^{2}+u_{2}^{2}\right)\right)^{n+1}} d u_{1} d u_{2}= \\
& =\frac{16 n}{\pi} \frac{4^{2 n}(2 n-1) ! c_{0}}{16^{n+1}} \int_{\mathbb{R}^{2}} \frac{1}{\left(\frac{\tau^{2}}{16}+t^{2}+\left(u_{1}^{2}+u_{2}^{2}\right)\right)^{n+1}} d u_{1} d u_{2}= \\
& =\frac{16 n}{\pi} \frac{4^{2 n}(2 n-1) ! c_{0}}{16^{n+1}} \int_{\frac{-\pi}{2}}^{\frac{3}{2} \pi} \int_{0}^{\infty} \frac{\rho}{\left(\frac{\tau^{2}}{16}+t^{2}+\rho^{2}\right)^{n+1}} d \rho d \theta= \\
& =\frac{4^{2 n}(2 n-1) ! c_{0}}{\left(\tau^{2}+16|z|^{2}\right)^{n}},
\end{aligned}
$$

where $z=t \xi$. Let $\varphi(\tau, z)=\frac{4^{2 n}(2 n-1) ! c_{0}}{\left(\tau^{2}+16|z|^{2}\right)^{n}}$. Now from the expression of the fundamental solution of $L$ in the classical case (see for example 4.3 of $[\mathrm{G}-\mathrm{S}(2)]$ ) we know that

$$
\varphi(\tau, t \xi)=\sum_{k \geq 0} \frac{(-1)}{2 k+2 n} \int_{-\infty}^{\infty} e^{i \lambda t} L_{k}^{2 n-1}\left(\frac{\lambda}{2}|\tau|\right) e^{-\frac{\lambda}{4}|\tau|}|\lambda|^{2 n-1} d \lambda .
$$

The first step to compute $\Phi$ is to change to polar coordinates in $\mathbb{R}^{3}$ the expression given in (1.4):

$$
\begin{array}{r}
<\Phi, f>=\sum_{k \in \mathbb{Z}_{\mathbb{R}^{3}}} \frac{1}{-|\lambda|(2 k+2(p-q))}<\varphi_{w, k}, f>|w|^{2 n} d w= \\
\quad=\sum_{k \in \mathbb{Z}_{S^{2}}} \int_{0}^{\infty} \frac{1}{-|\lambda|(2 k+2(p-q))}<\varphi_{\lambda \xi, k}, f>|\lambda|^{2 n+2} d \lambda d \xi .
\end{array}
$$

From the absolute convergence of (1.4) we can interchange the summation symbol with the integral 
over $S^{2}$. Because of $\triangle e^{i \lambda<\xi, z>}=-|\lambda|^{2} e^{i \lambda<\xi, z>}$, we have that

$$
\begin{aligned}
<\Phi & f>=\int_{S^{2}} \sum_{k \in \mathbb{Z}} \frac{(-1)}{(2 k+2(p-q))} \int_{0}^{\infty} \int_{N(p, q, \mathbb{H})} e^{i \lambda<\xi, z>} \theta_{\lambda, k}(\alpha) f(\alpha, z) d \alpha d z|\lambda|^{2 n+1} d \lambda d \xi= \\
& =\int_{S^{2}} \sum_{k \in \mathbb{Z}} \frac{1}{(2 k+2(p-q))} \int_{0}^{\infty} \int_{N(p, q, \mathbb{H})} \triangle e^{i \lambda<\xi, z>} \theta_{\lambda, k}(\alpha) f(\alpha, z) d \alpha d z|\lambda|^{2 n-1} d \lambda d \xi= \\
& =\int_{S^{2}} \sum_{k \in \mathbb{Z}} \frac{1}{(2 k+2(p-q))} \int_{0}^{\infty}<\varphi_{\lambda \xi, k}, \triangle f>|\lambda|^{2 n-1} d \lambda d \xi .
\end{aligned}
$$

Next we break the summation indexes according to $k \geq 2 q, k \leq-2 p$ and $-2 p<k<2 q$ to get the splitting $<\Phi, f>=<\Phi_{1}, f>+<\Phi_{2}, f>$, and as in section we change summation index to get the series starting from $k=0$. From the explicit definition of $\varphi_{\lambda \xi, k}$ we can write

$$
\begin{aligned}
<\Phi_{1}, f & >=\int_{S^{2}} \sum_{k \geq 0} \frac{1}{2 k+2 n} \int_{0}^{\infty} \int_{\mathbb{R}^{3}} e^{i \lambda<\xi, z>} \times \\
& \times<T_{\lambda, k+2 q}-T_{\lambda,-k-2 p}, N \triangle f(., z)>d z|\lambda|^{2 n-1} d \lambda d \xi
\end{aligned}
$$

where $T_{\lambda, k}$ is defined by equations (1.6) and (1.7). By performing similar computations than in section 2 and introducing the function

$$
G_{f}(\tau, z)=N f(\tau, z)-\sum_{j=0}^{2 n-2} \frac{\partial^{j} N f}{\partial \tau^{j}}(0, z) \frac{\tau^{j}}{j !}
$$

we get the splitting $\left\langle\Phi_{1}, f>=<\Phi_{11}, f>+<\Phi_{12}, f>\right.$, where

$$
\begin{aligned}
&<\Phi_{11}, f>=\int_{S^{2}} \sum_{k \geq 0} \frac{(-1)}{2 k+2 n} \int_{0}^{\infty} \int_{\mathbb{R}^{3}} \int_{-\infty}^{\infty} e^{i \lambda<\xi, z>} \times \\
& \times \operatorname{sgn}(\tau) L_{k}^{2 n-1}\left(\frac{2}{\lambda}|\tau|\right) e^{-\frac{\lambda}{4}|\tau|} \triangle G_{f}(\tau, z) d \tau d z|\lambda|^{2 n-1} d \lambda d \xi, \\
&<\Phi_{12}, f>=2 \int_{S^{2}} \sum_{k \geq 0} \frac{1}{2 k+2 n} \int_{0}^{\infty} \int_{\mathbb{R}^{3}} e^{i \lambda<\xi, z>} \times \\
& \times \sum_{\substack{l=0 \\
l \text { odd }}}^{2 n-2}\left(\frac{2}{\lambda}\right)^{l+1}\left(a_{k l}+b_{k l}\right)<\delta^{(l)}, \triangle N f(., z)>d z|\lambda|^{2 n-1} d \xi,
\end{aligned}
$$

and $a_{k l}, b_{k l}$ are the same constant defined in (2.8) and (2.3), respectively. Now let us recall the fact that

$$
\int_{S^{2}} \int_{0}^{\infty} e^{i \lambda<\xi, z>} F(|\lambda|) d \lambda d \xi=\frac{1}{2} \int_{S^{2}} \int_{-\infty}^{\infty} e^{i \lambda<\xi, z>} F(|\lambda|) d \lambda d \xi,
$$


and we apply the dual Radon transform to (3.2).

Observe now that

$$
\int_{-\infty}^{\infty} \int_{\mathbb{R}^{3}} \frac{\operatorname{sgn}(\tau) G_{f}(\tau, z)}{\left(1+16|z|^{2}\right)^{n+1}} d z d \tau
$$

converges, which we can see by changing to polar variables in $\mathbb{R}^{3}$ and arguing like in lemma 2.2 of $[\mathrm{G}-\mathrm{S}(2)]$.

We finally get that

$$
\begin{gathered}
<\Phi_{11}, f>=\frac{1}{2}<-2 \pi \frac{16 n}{\pi} \frac{4^{2 n}(2 n-1) ! c_{0}}{\left(\tau^{2}+16|z|^{2}\right)^{n+1}}, \operatorname{sgn}(\tau) G_{f}(\tau, z)>= \\
=-4^{2 n+2} n(2 n-1) ! c_{0}<\frac{1}{\left(\tau^{2}+16|z|^{2}\right)^{n+1}}, \operatorname{sgn}(\tau) G_{f}(\tau, z)>.
\end{gathered}
$$

We have thus proven that the expression defining $\Phi_{11}$ is finite. Then the expression defining $\Phi_{12}$ is also finite, and by Abel's lemma we can write

$$
<\Phi_{12}, f>=2 \lim _{r \rightarrow 1^{-}} \lim _{\epsilon \rightarrow 0^{+}} \sum_{k \geq 0} \sum_{\substack{l=0 \\ l \text { odd }}}^{2 n-2} 2^{l+1}\left(a_{k l}+b_{k l}\right) \frac{r^{2 k+2 n}}{2 k+2 n} d_{\epsilon, l, f},
$$

where

$$
d_{\epsilon, l, f}=\int_{S^{2}} \int_{0}^{\infty} \int_{\mathbb{R}^{3}} e^{-\epsilon \lambda} e^{i \lambda<\xi, z>}|\lambda|^{2 n-l-2}<\delta^{(l)}, \triangle N f(., z)>d z d \lambda d \xi .
$$

We need to compute $\lim _{\epsilon \rightarrow 0^{+}} d_{\epsilon, l, f}$. Observing that $\triangle e^{i \lambda<\xi, z>}=-|\lambda|^{2} e^{i \lambda<\xi, z>}$, we have that

$$
\begin{aligned}
d_{\epsilon, l, f} & =(-1)^{l+1} \int_{S^{2}} \int_{0}^{\infty} \int_{\mathbb{R}^{3}} e^{-\epsilon \lambda} e^{i \lambda<\xi, z>}|\lambda|^{2 n-l} \frac{\partial^{l}}{\partial \tau^{l}} N f(0, z) d z d \lambda d \xi= \\
& =(-1)^{l+1} \int_{\mathbb{R}^{3}} \int_{\mathbb{R}^{3}} e^{-\epsilon|x|}|x|^{2 n-l-2} e^{i<x, z>} \frac{\partial^{l}}{\partial \tau^{l}} N f(0, z) d z d x,
\end{aligned}
$$

where we have changed to cartesian coordinates in $\mathbb{R}^{3}$. To solve this integral let us observe that

$$
(-1)^{2 n-l-2} e^{-\epsilon|x|}|x|^{2 n-l-2}=\frac{\partial^{2 n-l-2}}{\partial \epsilon^{2 n-l-2}} P_{\epsilon}(x),
$$

where $P_{\epsilon}(x)$ is the Poisson kernel and ^denotes the Fourier transform. Let us write

$$
d_{\epsilon, l, f}=(-1)^{l} \int_{\mathbb{R}^{3}} \frac{\partial^{2 n-l-2}}{\partial \epsilon^{2 n-l-2}} P_{\epsilon}(x)\left(\frac{\partial^{l}}{\partial \tau^{l}} N f(0, .)\right) \hat{(x)} d x=(-1)^{l} \frac{\partial^{2 n-l-2}}{\partial \epsilon^{2 n-l-2}}\left(P_{\epsilon} * h\right)(0) .
$$

Taking limit as $\epsilon \rightarrow 0^{+}$we obtain

$$
\lim _{\epsilon \rightarrow 0^{+}}=(-1)(-\triangle)^{\frac{2 n-l-2}{2}} \frac{\partial^{l}}{\partial \tau^{l}} N f(0,0)
$$


where $(-\triangle)^{\frac{2 n-l-2}{2}}$ is a fractionary exponential of the Laplacian (see for example [S-Sh]), which is the operator defined for $g \in \mathcal{S}\left(\mathbb{R}^{3}\right)$ by

$$
(-\triangle)^{\frac{2 n-l-2}{2}} g(x)=\int_{\mathbb{R}^{3}}|\omega|^{2 n-l-2} \widehat{g}(\omega) e^{i<\omega, z>} d \omega .
$$

This computation together with proposition 4.8 of [G-S(2)] lets us write

$$
<\Phi_{12}, f>=\sum_{\substack{l=0 \\
l \text { odd }}}^{2 n-2} \sum_{j=1}^{l+1} \frac{1}{2^{2 n-2 l-j-3}} c_{j}\left(\begin{array}{c}
2 n-j-1 \\
l-j-1
\end{array}\right)(-1)(-\triangle)^{\frac{2 n-l-2}{2}} \frac{\partial^{l}}{\partial \tau^{l}} N f(0,0),
$$

where each $c_{j}$ is the constant defined in Remark 4.7 of [G-S $\left.(2)\right]$.

After performing the usual computations for $\Phi_{2}$ we have proved the main theorem of this section:

Theorem 3.1. Let $c_{0}$ and $c_{j}$ be the constants defined above. Then

$$
\begin{aligned}
& <\Phi, f>=-4^{2 n+2} n(2 n-1) ! c_{0}<\frac{1}{\left(\tau^{2}+16|z|^{2}\right)^{n+1}}, \operatorname{sgn}(\tau) G_{f}(\tau, z)>+ \\
& +\sum_{\substack{l=0 \\
l \text { odd }}}^{2 n-2} \sum_{j=1}^{l+1} \frac{1}{2^{2 n-2 l-j-3}} c_{j}\left(\begin{array}{c}
2 n-j-1 \\
l-j-1
\end{array}\right)(-1)(-\triangle)^{\frac{2 n-l-2}{2}} \frac{\partial^{l}}{\partial \tau^{l}} N f(0,0)+ \\
& +\sum_{-2 q<k<0} \frac{1}{2 k+2(p-q)}\left[(-1)^{k+1} \sum_{r=0}^{k+2 p-1}\left(\begin{array}{c}
k+2 p-1 \\
r
\end{array}\right) 2^{-k-2 p+2 r+2} \times\right. \\
& \times(-1)(-\triangle)^{\frac{2 n-r-2}{2}} \frac{\partial^{r}}{\partial \tau^{r}} N f(0,0)+ \\
& +\sum_{l=0}^{2 n-2-k-2 p}(-1)^{-l-k}\left(\begin{array}{c}
-k-2 p+2 n-1 \\
2 n-2-l-k-2 p
\end{array}\right) \sum_{r=0}^{l+k-2 p}\left(\begin{array}{c}
l+k-2 p \\
r
\end{array}\right) \times \\
& \left.\times(-1) 2^{-l-k+2 p+2 r+1}(-\triangle)^{\frac{2 n-r-2}{2}} \frac{\partial^{r}}{\partial \tau^{r}} N f(0,0)\right]+ \\
& +\sum_{0 \leq k<2 q} \frac{1}{2 k+2(p-q)}\left[(-1)^{k+1} \sum_{r=0}^{-k+2 q-1}\left(\begin{array}{c}
-k+2 q-1 \\
r
\end{array}\right) \times\right. \\
& \times 2^{k-2 q+2 r+1}(-1)^{r}(-\triangle)^{\frac{2 n-r-2}{2}} \frac{\partial^{r}}{\partial \tau^{r}} N f(0,0)+ \\
& +\sum_{l=0}^{2 n-2+k-2 q}(-1)^{-l+k-2 q}\left(\begin{array}{c}
k-2 q+2 n-1 \\
2 n-2-l+k-2 q
\end{array}\right) \sum_{r=0}^{l-k+2 q}\left(\begin{array}{c}
l-k+2 q \\
r
\end{array}\right) \times \\
& \left.\times 2^{-l+k-2 q+2 r+1}(-1)^{r}(-\triangle)^{\frac{2 n-r-2}{2}} \frac{\partial^{r}}{\partial \tau^{r}} N f(0,0)\right] .
\end{aligned}
$$

\section{Remark}

Let $N$ be a group of Heisenberg type and let $\eta$ be its Lie algebra. So $\eta=V \oplus \mathfrak{z}$, with $\operatorname{dim} V=2 m$ and $\operatorname{dim} \mathfrak{z}=k$. Let $U(V)$ be the unitary group acting on $V$. Then it is known $([\mathrm{R}])$ that $(N \ltimes U(V), U(V))$ 
is a Gelfand pair, and also in $[\mathrm{R}]$ were computed the spherical functions. We fix an orthonormal basis of $V,\left\{X_{1}, \ldots, X_{2 m}\right\}$, and consider the operator

$$
L=\sum_{j=1}^{2 m} X_{j}^{2} .
$$

With the same arguments as above, using the Radon transform in $\mathbb{R}^{k}$ and the fundamental solution of $L$ in the classical Heisenberg group $2 m+1$ dimensional, we can recover the fundamental solution of $L$ (see $[\mathrm{K}],[\mathrm{R}])$.

\section{References}

[]B-D-R C. Benson, A.H. Dooley and G. Ratcliff, Fundamental solutions for powers of the Heisenberg sub-Laplacian, Ill. J. Math. 37, No.3, 455-476 (1993).

[]D-M G. Van Dijk and K. Mokni, Harmonic analysis on a class of generalized Gel'fand pairs associated with hyperbolic spaces, Russ. J. Math. Phys. 5, No.2, 167-178 (1997).

[]F G.B. Folland A fundamental solution for a subelliptic operator, Bull. Amer. Math. Soc. 79 (1973), 373-376.

[]F-S G.B. Folland and E.M. Stein, Estimates for the $\bar{\partial}_{b}$ complex and analysis on the Heisenberg group., Commun. Pure Appl. Math. 27, 429-522 (1974).

[]G-S(1) T. Godoy, L. Saal, $L^{2}$ spectral decomposition on the Heisenberg group associated to the action of $U(p, q)$, Pac. J. Math. 193, No.2, 327-353 (2000).

[]G-S(2) T. Godoy, L. Saal, On the relative fundamental solutions for a second order differential operator on the Heisenberg group, Stud. Math. 145, No.2, 143-164 (2001).

[]K A. Kaplan, Fundamental solutions for a class of hypoelliptic PDE generated by composition of quadratic forms, Trans. Am. Math. Soc. 258, 147-153 (1980).

[]K-R A. Kaplan and F. Ricci, Harmonic analysis on groups of Heisenberg type, Lecture notes on Math 992, 1983, 416-435.

[]M-R D. Müller and F. Ricci, On the Laplace-Beltrami operator on the oscillator group, J. Reine Angew. Math. 390, 193-207 (1988).

[]R F. Ricci, Harmonic analysis on groups of type H, preprint.

[]S-Sh E.M. Stein and R. Shakarchi, Fourier Analysis:An Introduction, Princeton University. Press, 2003.

[]T A. Tengstrand, Distributions invariant under an orthogonal group of arbitrary signature, Math. Scand. 8, 201-218 (1960). 
[]V R. Vidal, Anlisis armnico en el grupo de Heisenberg cuaterninico asociado al grupo Spin $(3) \times$ $U(p, q, \mathbb{H})$, Trabajos de Matemtica - FaMAF, 2009. 